\title{
ON THE INVARIANT THEORY FOR ACYCLIC GENTLE ALGEBRAS
}

\author{
ANDREW T. CARROLL AND CALIN CHINDRIS
}

\begin{abstract}
In this paper we show that the fields of rational invariants over the irreducible components of the module varieties for an acyclic gentle algebra are purely transcendental extensions. Along the way, we exhibit for such fields of rational invariants a transcendence basis in terms of Schofield's determinantal semi-invariants.

We also show that moduli spaces of modules over regular irreducible components are just products of projective spaces.
\end{abstract}

\section{Contents}

1. Introduction

2. Background

3. Rational invariants

4. Moduli spaces of modules

5. Acyclic gentle algebras

6. Proofs of the main results

7. Concluding remarks

Acknowledgements

\section{INTRODUCTION}

Throughout the article, $k$ always denotes an algebraically closed field of characteristic zero. All algebras (associative and with identity) are assumed to be finite-dimensional over $k$, and all modules are assumed to be finite-dimensional left modules.

One of the fundamental problems in the representation theory of algebras is that of classifying the indecomposable modules. Based on the complexity of these modules, one distinguishes the class of tame algebras and that of wild algebras. According to the remarkable Tame-Wild Dichotomy Theorem of Drozd [26], these two classes of algebras are disjoint and cover the whole class of algebras. Since the representation theory of a wild algebra is at least as complicated as that of a free algebra in two variables, and since the later theory is known to be undecidable, one can hope to meaningfully classify the indecomposable modules only for tame

Received by the editors October 12, 2012 and, in revised form, May 10, 2013 and May 18, 2013.

2010 Mathematics Subject Classification. Primary 16G10, 16G60, 16R30.

Key words and phrases. Gentle algebras, module varieties, moduli spaces of modules, rank sequences, rational invariants, up and down graphs.

The second author was supported by NSF grant DMS-1101383. 
algebras. For more precise definitions, see [39, Chapter XIX] and the references therein.

An interesting task in the representation theory of algebras is to study, for a given finite-dimensional algebra, the geometry of the affine varieties of modules of fixed dimension vectors and the actions of the corresponding products of general linear groups. In particular, it would be interesting to find characterizations of prominent classes of tame algebras via geometric properties of their module varieties. This research direction has attracted much attention during the last two decades (see for example [4], [5], 6], 7], [13, [23], 27], 33], 34, 35], 40]).

In this paper, we seek characterizations of tame algebras in terms of invariant theory. A first result in this direction was obtained by Skowroński and Weyman in [40. Theorem 1], where they showed that a finite-dimensional algebra of global dimension one is tame if and only if all of its algebras of semi-invariants are complete intersections. Unfortunately, this result does not extend to algebras of higher global dimension (not even of global dimension two), as shown by Kraśkiewicz in [29. As it was suggested by Weyman, in order to characterize the tameness of an algebra via invariant theory, one should impose geometric conditions on the various moduli spaces of semi-stable modules rather than on the entire algebras of semi-invariants. For more precise details, see Section 7

A description of the tameness of quasi-tilted algebras in terms of the invariant theory of the algebras in question has been found in [14,15. In this paper, we continue this line of inquiry for the class of triangular gentle algebras, which are known to be tame. Their indecomposable modules can be nicely classified, however these algebras still represent an increase in the level of complexity from the tame quasi-tilted case. For example, it is possible to construct triangular gentle algebras of arbitrarily large global dimension. Furthermore, the number of one-parameter families required to parameterize $d$-dimensional indecomposable modules can grow faster than any polynomial in $d$.

Theorem 1. Let $A=k Q / I$ be a triangular gentle algebra, $\mathbf{d}$ a dimension vector of $A$, and $C \subseteq \bmod (A, \mathbf{d})$ an irreducible component. Then the following statements hold:

(1) the field of rational invariants $k(C)^{\mathrm{GL}(d)}$ is a purely transcendental extension of $k$ whose transcendece degree equals the sum of the multiplicities of the indecomposable irreducible regular components occurring in the generic decomposition of $C$;

(2) if $C$ is an irreducible regular component, then for any weight $\theta \in \mathbb{Z}^{Q_{0}}$ with $C_{\theta}^{s s} \neq \emptyset$ and such that the $\theta$-stable summands of $C$ are regular, the moduli space $\mathcal{M}(C)_{\theta}^{s s}$ is just a product of projective spaces.

Our next main result gives a transcendence basis for $k(C)^{\mathrm{GL}(\mathbf{d})}$ when $C$ is an irreducible regular component. We do this via the so-called up and down graphs, introduced in the context of triangular gentle algebras by the first author (see [11]). The up and down graphs are combinatorially defined objects which hold valuable geometric information in that they: (1) give the generic decomposition of irreducible components in module varieties; (2) explicitly describe the generic modules in irreducible components; and (3) allow for explicit computations of the so-called generalized Schofield's semi-invariants which in turn are rather remarkable coordinates on moduli spaces of modules for finite-dimensional algebras. 
Theorem 2. Let $A=k Q / I$ be a triangular gentle algebra, $\bmod (A, \mathbf{d}, \mathbf{r})$, be an irreducible regular component, and

$$
\bmod (A, \mathbf{d}, \mathbf{r})=\overline{\bigoplus_{i=1}^{n} \bmod \left(A, \mathbf{d}_{i}, \mathbf{r}_{i}\right)^{\oplus m_{i}}}
$$

be the generic decomposition of $\bmod (A, \mathbf{d}, \mathbf{r})$ where $\bmod \left(A, \mathbf{d}_{i}, \mathbf{r}_{i}\right), 1 \leq i \leq n$, are (pairwise distinct) indecomposable irreducible regular components and $m_{1}, \ldots, m_{n}$ are positive integers.

For each $i$, let $\lambda(i, j), 0 \leq j \leq m_{i}$, be pairwise distinct elements of $k^{*}$ and $M\left(\mathbf{d}_{i}, \mathbf{r}_{i}, \lambda(i, j)\right), 0 \leq j \leq m_{i}$, be the corresponding generic modules of $\bmod \left(A, \mathbf{d}_{i}, \mathbf{r}_{i}\right)$. Then, $k(\bmod (A, \mathbf{d}, \mathbf{r}))^{\mathrm{GL}(\mathbf{d})}$ is a purely transcendental extension of $k$ of degree $\sum_{i=1}^{n} m_{i}$ with transcendence basis

$$
\left\{\frac{\bar{c}^{M\left(\mathbf{d}_{i}, \mathbf{r}_{i}, \lambda(i, j)\right)}}{\bar{c}^{M\left(\mathbf{d}_{i}, \mathbf{r}_{i}, \lambda(i, j+1)\right)}} ; i=1, \ldots, n, j=0, \ldots, m_{i}-1\right\} .
$$

We point out that the class of tame hereditary algebras is the only other class of tame algebras for which a similar transcendence basis has been constructed (see [36]). For wild quivers, the corresponding rationality problem, which has been open for more than 45 years, is one of the most important open problems in rational invariant theory (see [8]). It is our hope that the representation-theoretic description of the transcendence basis in the theorem above will inspire the construction of transcendence bases in other situations, too.

In Section 2, we outline the pertinent notions related to bound quiver algebras. This includes a description of the module varieties and generic decomposition of irreducible components due to Crawley-Boevey and Schröer. Section 3 recalls the basic notions of rational invariants and rational quotients. It includes a general reduction result for fields of rational invariants over irreducible components in module varieties. In Section 4, we first review King's construction of moduli spaces of modules for finite-dimensional algebras, and then state another general reduction result that allows one to break a moduli space of modules into products of smaller ones. We also show that, at least in the tame case, these smaller moduli spaces are rather well-behaved (see Proposition 7). These general results are applied to gentle algebras in Section [5] following a recollection of the construction of their generic modules. We prove a number of facts about these irreducible components which allow an application of the reduction techniques of Sections 4 and 3 . In Section 6 we prove the two main theorems of the paper, and point out some idiosynchracies that arise in the generic decompositions of modules over gentle algebras. Section 7 concludes the article by placing the results in the framework of an effort to characterize tameness of an algebra via invariant theoretic characteristics.

\section{BACKGROUND}

2.1. Bound quiver algebras. Let $Q=\left(Q_{0}, Q_{1}, t, h\right)$ be a finite quiver with vertex set $Q_{0}$ and arrow set $Q_{1}$. The two functions $t, h: Q_{1} \rightarrow Q_{0}$ assign to each arrow $a \in Q_{1}$ its tail $t a$ and head $h a$, respectively.

A representation $V$ of $Q$ over $k$ is a collection $(V(x), V(a))_{x \in Q_{0}, a \in Q_{1}}$ of finitedimensional $k$-vector spaces $V(x), x \in Q_{0}$, and $k$-linear maps $V(a) \in$ $\operatorname{Hom}_{k}(V(t a), V(h a)), a \in Q_{1}$. The dimension vector of a representation $V$ of $Q$ is the function $\operatorname{dim} V: Q_{0} \rightarrow \mathbb{Z}$ defined by $(\operatorname{dim} V)(x)=\operatorname{dim}_{k} V(x)$ for $x \in Q_{0}$. 
Let $S_{x}$ be the one-dimensional representation of $Q$ at vertex $x \in Q_{0}$. By a dimension vector of $Q$, we simply mean a vector $\mathbf{d} \in \mathbb{Z}_{\geq 0}^{Q_{0}}$.

Given two representations $V$ and $W$ of $Q$, we define a morphism $\varphi: V \rightarrow W$ to be a collection $(\varphi(x))_{x \in Q_{0}}$ of $k$-linear maps with $\varphi(x) \in \operatorname{Hom}_{k}(V(x), W(x))$ for each $x \in Q_{0}$, and such that $\varphi(h a) V(a)=W(a) \varphi(t a)$ for each $a \in Q_{1}$. We denote by $\operatorname{Hom}_{Q}(V, W)$ the $k$-vector space of all morphisms from $V$ to $W$. Let $V$ and $W$ be two representations of $Q$. We say that $V$ is a subrepresentation of $W$ if $V(x)$ is a subspace of $W(x)$ for each $x \in Q_{0}$ and $V(a)$ is the restriction of $W(a)$ to $V(t a)$ for each $a \in Q_{1}$. In this way, we obtain the abelian category $\operatorname{rep}(Q)$ of all representations of $Q$.

Given a quiver $Q$, its path algebra $k Q$ has a $k$-basis consisting of all paths (including the trivial ones) and the multiplication in $k Q$ is given by concatenation of paths. It is easy to see that any $k Q$-module defines a representation of $Q$, and vice versa. Furthermore, the category $\bmod (k Q)$ of $k Q$-modules is equivalent to the category $\operatorname{rep}(Q)$. In what follows, we identify $\bmod (k Q)$ and $\operatorname{rep}(Q)$, and use the same notation for a module and the corresponding representation.

A two-sided ideal $I$ of $k Q$ is said to be admissible if there exists an integer $L \geq 2$ such that $R_{Q}^{L} \subseteq I \subseteq R_{Q}^{2}$. Here, $R_{Q}$ denotes the two-sided ideal of $k Q$ generated by all arrows of $Q$.

If $I$ is an admissible ideal of $k Q$, the pair $(Q, I)$ is called a bound quiver and the quotient algebra $k Q / I$ is called the bound quiver algebra of $(Q, I)$. Any admissible ideal is generated by finitely many admissible relations, and any bound quiver algebra is finite-dimensional and basic. Moreover, a bound quiver algebra $k Q / I$ is connected if and only if (the underlying graph of) $Q$ is connected (see for example [3. Lemma II.2.5]).

Up to Morita equivalence, any finite-dimensional algebra $A$ can be viewed as the bound quiver algebra of a bound quiver $\left(Q_{A}, I\right)$, where $Q_{A}$ is the Gabriel quiver of $A$ (see [3, Corollary I.6.10 and Theorem II.3.7]). (Note that the ideal of relations $I$ is not uniquely determined by $A$.) We say that $A$ is a triangular algebra if its Gabriel quiver has no oriented cycles.

Fix a bound quiver $(Q, I)$ and let $A=k Q / I$ be its bound quiver algebra. A representation $M$ of $A$ (or $(Q, I)$ ) is just a representation $M$ of $Q$ such that $M(r)=0$ for all $r \in I$. The category $\bmod (A)$ of finite-dimensional left $A$-modules is equivalent to the category $\operatorname{rep}(A)$ of representations of $A$. As before, we identify $\bmod (A)$ and $\operatorname{rep}(A)$, and make no distinction between $A$-modules and representations of $A$. For each vertex $x \in Q_{0}$, we denote by $P_{x}$ the indecomposable projective cover of the simple $A$-module $S_{x}$. For an $A$-module $M$, we denote by $\operatorname{pdim} M$ its projective dimension. An $A$-module $M$ is called $S$ chur if $\operatorname{End}_{A}(M) \cong k$.

An algebra $A$ is called tame if for each dimension vector $\mathbf{d} \in \mathbb{Z}_{\geq 0}^{Q_{0}}$, the subcategory $\operatorname{ind}_{\mathbf{d}}(A)$, whose objects are the indecomposable d-dimensional $A$-modules, is parametrized, the sense of [39], by a finite family of functors $F_{i}:=Q_{i} \otimes_{A_{i}}-$ : $\operatorname{ind}_{1}\left(A_{i}\right) \rightarrow \bmod (A), 1 \leq i \leq n$, where for each $1 \leq i \leq n$, we have: (1) $A_{i}=k$ or $A_{i}=k[t]_{h_{i}}$ is a localization of $k[t] ;(2) Q_{i}$ is an $A-A_{i}$-bimodule which is finitely generated and free as a right $A_{i}$-module; and (3) the functor $F_{i}$ is a representation embedding. For more details, we refer to [24] and [39].

For the remainder of this subsection, we assume that $A$ has finite global dimension; this happens, for example, when $Q$ has no oriented cycles. The Euler form of 
$A$ is the bilinear form $\langle\langle\cdot, \cdot\rangle\rangle_{A}: \mathbb{Z}^{Q_{0}} \times \mathbb{Z}^{Q_{0}} \rightarrow \mathbb{Z}$ defined by

$$
\langle\langle\mathbf{d}, \mathbf{e}\rangle\rangle_{A}=\sum_{l \geq 0}(-1)^{l} \sum_{x, y \in Q_{0}} \operatorname{dim}_{k} \operatorname{Ext}_{A}^{l}\left(S_{x}, S_{y}\right) \mathbf{d}(x) \mathbf{e}(y) .
$$

Note that if $M$ is a $\mathbf{d}$-dimensional $A$-module and $N$ is an e-dimensional $A$-module, then

$$
\langle\langle\mathbf{d}, \mathbf{e}\rangle\rangle_{A}=\sum_{l \geq 0}(-1)^{l} \operatorname{dim}_{k} \operatorname{Ext}_{A}^{l}(M, N) .
$$

2.2. Module varieties and their irreducible components. Let $\mathbf{d}$ be a dimension vector of $A=k Q / I$ (or equivalently, of $Q$ ). The affine variety

$$
\bmod (A, \mathbf{d}):=\left\{M \in \prod_{a \in Q_{1}} \operatorname{Mat}_{\mathbf{d}(h a) \times \mathbf{d}(t a)}(k) \mid M(r)=0, \forall r \in I\right\}
$$

is called the module/representation variety of $\mathbf{d}$-dimensional modules/representations of $A$. The affine $\operatorname{space} \bmod (Q, \mathbf{d}):=\prod_{a \in Q_{1}} \operatorname{Mat}_{\mathbf{d}(h a) \times \mathbf{d}(t a)}(k)$ is acted upon by the base change group

$$
\mathrm{GL}(\mathbf{d}):=\prod_{x \in Q_{0}} \mathrm{GL}(\mathbf{d}(x), k)
$$

by simultaneous conjugation, i.e., for $g=(g(x))_{x \in Q_{0}} \in \mathrm{GL}(\mathbf{d})$ and $V=(V(a))_{a \in Q_{1}}$ $\in \bmod (Q, \mathbf{d}), g \cdot V$ is defined by

$$
(g \cdot V)(a)=g(h a) V(a) g(t a)^{-1}, \forall a \in Q_{1} .
$$

It can be easily seen that $\bmod (A, \mathbf{d})$ is a $\mathrm{GL}(\mathbf{d})$-invariant closed subvariety of $\bmod (Q, \mathbf{d})$, and that the $\mathrm{GL}(\mathbf{d})$ - orbits in $\bmod (A, \mathbf{d})$ are in one-to-one correspondence with the isomorphism classes of the $\mathbf{d}$-dimensional $A$-modules. Note that $\bmod (A, \mathbf{d})$ does not have to be irreducible.

Let $C$ be an irreducible component of $\bmod (A, \mathbf{d})$. We say that $C$ is indecomposable if $C$ has a non-empty open subset of indecomposable modules. We say that $C$ is a Schur component if $C$ contains a Schur module. Note that any Schur component is indecomposable. Whenever $\bmod (A, \mathbf{d})$ has an indecomposable irreducible component, we say that $\mathbf{d}$ is a generic root of $A$.

Given a decomposition $\mathbf{d}=\mathbf{d}_{1}+\ldots+\mathbf{d}_{t}$, where $\mathbf{d}_{i} \in \mathbb{Z}_{\geq 0}^{Q_{0}}, 1 \leq i \leq t$, and $\mathrm{GL}\left(\mathbf{d}_{i}\right)$-invariant constructible subsets $C_{i} \subseteq \bmod \left(A, \mathbf{d}_{i}\right), 1 \leq i \leq t$, we denote by $C_{1} \oplus \ldots \oplus C_{t}$ the constructible subset of $\bmod (A, \mathbf{d})$ defined as

$$
C_{1} \oplus \ldots \oplus C_{t}=\left\{M \in \bmod (A, \mathbf{d}) \mid M \simeq \bigoplus_{i=1}^{t} M_{i} \text { with } M_{i} \in C_{i}, \forall 1 \leq i \leq t\right\} .
$$

As shown by de la Peña in [20, Section 1.3] and Crawley-Boevey and Schröer in [17, Theorem 1.1], any irreducible component of a module variety satisfies a Krull-Schmidt type decomposition. Specifically, if $C$ is an irreducible component of $\bmod (A, \mathbf{d})$, then there are unique generic roots $\mathbf{d}_{1}, \ldots, \mathbf{d}_{t}$ of $A$ such that $\mathbf{d}=$ $\mathbf{d}_{1}+\ldots+\mathbf{d}_{t}$ and

$$
C=\overline{C_{1} \oplus \ldots \oplus C_{t}}
$$

for some indecomposable irreducible components $C_{i} \subseteq \bmod \left(A, \mathbf{d}_{i}\right), 1 \leq i \leq t$. Moreover, the indecomposable irreducible components $C_{i}, 1 \leq i \leq t$, are uniquely determined by this property. We call $\mathbf{d}=\mathbf{d}_{1} \oplus \ldots \oplus \mathbf{d}_{t}$ the generic decomposition of $\mathbf{d}$ in $C$, and $C=\overline{C_{1} \oplus \ldots \oplus C_{t}}$ the generic decomposition of $C$. 
It also follows from [17, Theorem 1.2] that if $C=\overline{C_{1} \oplus \ldots \oplus C_{t}}$ is the generic decomposition of $C \subseteq \bmod (A, \mathbf{d})$, then $\overline{\bigoplus_{i \in J} C_{i}}$ is an irreducible component of $\bmod \left(A, \sum_{i \in J} \mathbf{d}_{i}\right)$ for any subset $J \subseteq\{1, \ldots, t\}$.

For the remainder of this section, we assume that $A$ is a tame algebra and let $\mathbf{d}$ be a generic root of $A$. Denote by $\operatorname{ind}(A, \mathbf{d})$ the constructible subset of $\bmod (A, \mathbf{d})$ consisting of all $\mathbf{d}$-dimensional indecomposable $A$-modules.

We know from the work of Dowbor and Skowroński in 24 that there are finitely many principal open subsets $\mathcal{U}_{i} \subseteq \mathbb{A}^{1}=k$ and regular morphisms $f_{i}: \mathcal{U}_{i} \rightarrow$ $\bmod (A, \mathbf{d}), 1 \leq i \leq n$, such that:

- for each $1 \leq i \leq n, f_{i}\left(\mathcal{U}_{i}\right) \subset \operatorname{ind}(A, \mathbf{d})$, and if $f_{i}\left(\lambda_{1}\right) \simeq f_{i}\left(\lambda_{2}\right)$ as $A$-modules, then $\lambda_{1}=\lambda_{2}$;

- all modules in ind $(A, \mathbf{d})$, except possibly finitely many isoclasses, belong to $\bigcup_{i=1}^{n} \mathcal{F}_{i}$, where each $\mathcal{F}_{i}$ is the closure of the image of the action morphism $\operatorname{GL}(\mathbf{d}) \times \mathcal{U}_{i} \rightarrow \bmod (A, \mathbf{d})$ that sends $(g, \lambda)$ to $g \cdot f_{i}(\lambda)$, i.e. $\mathcal{F}_{i}=$ $\overline{\bigcup_{\lambda \in \mathcal{U}_{i}} \mathrm{GL}(\mathbf{d}) f_{i}(\lambda)}$.

(We call $\left(\mathcal{U}_{i}, f_{i}\right), 1 \leq i \leq n$, parameterizing pairs for $\operatorname{ind}(A, \mathbf{d})$.)

Consequently, we have that

$$
\overline{\operatorname{ind}(A, \mathbf{d})}=\bigcup_{i=1}^{n} \mathcal{F}_{i} \cup \bigcup_{j=1}^{l} \overline{\operatorname{GL}(\mathbf{d}) M_{j}},
$$

where $M_{1}, \ldots, M_{l} \in \operatorname{ind}(A, \mathbf{d})$.

Now, let $C \subseteq \bmod (A, \mathbf{d})$ be an indecomposable irreducible component; in particular, $C$ is an irreducible component of $\overline{\operatorname{ind}(A, \mathbf{d})}$. From the discussion above, it follows that either:

- $C=\mathcal{F}_{i}$ for some $1 \leq i \leq n$ or

- $C=\overline{\mathrm{GL}(\mathbf{d}) M_{j}}$ for some $1 \leq j \leq l$.

In the tame case, we have the following simple but very useful dimension count:

Lemma 3. Let $A=k Q / I$ be a tame bound quiver algebra, $\mathbf{d}$ a generic root, and $C \subseteq \bmod (A, \mathbf{d})$ an indecomposable irreducible component. Then,

$$
\operatorname{dim} C-\operatorname{dim} \operatorname{GL}(\mathbf{d})+\min \left\{\operatorname{dim}_{k} \operatorname{End}_{A}(M) \mid M \in C\right\} \in\{0,1\} .
$$

Proof. If $C$ is an orbit closure, then the expression above is obviously zero. The only other possibility is when $C=\overline{\bigcup_{\lambda \in \mathcal{U}} \mathrm{GL}(\mathbf{d}) f(\lambda)}$ with $(\mathcal{U}, f)$ a parameterizing pair as above. The action morphism $\mu: \mathrm{GL}(\mathbf{d}) \times \mathcal{U} \rightarrow C, \mu(g, \lambda)=g f(\lambda), \forall(g, \lambda) \in \mathrm{GL}(\mathbf{d}) \times$ $\mathcal{U}$, is dominant by construction and, moreover, for a generic $M_{0}=\mu\left(g_{0}, \lambda_{0}\right) \in C$ :

$$
\begin{aligned}
\mu^{-1}\left(M_{0}\right) & =\left\{(g, \lambda) \in \mathrm{GL}(\mathbf{d}) \times \mathcal{U} \mid g \cdot f(\lambda)=g_{0} \cdot f\left(\lambda_{0}\right)\right\} \\
& =g_{0} \operatorname{Stab}_{\mathrm{GL}(\mathbf{d})}\left(f\left(\lambda_{0}\right)\right) \times\left\{\lambda_{0}\right\} .
\end{aligned}
$$

In particular, this shows that $\operatorname{dim} \mu^{-1}\left(M_{0}\right)=\operatorname{dim}_{k} \operatorname{End}_{A}\left(M_{0}\right)$. Now using the theorem on the generic fiber, we get that $\operatorname{dim} \mathrm{GL}(\mathbf{d})+1-\operatorname{dim} C=\operatorname{dim}_{k} \operatorname{End}_{A}\left(M_{0}\right)$, which is exactly what we need to prove.

\section{RATIONAL INVARIANTS}

Let $A=k Q / I$ be a bound quiver algebra, $\mathbf{d} \in \mathbb{Z}_{\geq 0}^{Q_{0}}$ a dimension vector of $A$, and $C$ a $\mathrm{GL}(\mathbf{d})$-invariant irreducible closed subset of $\bmod (A, \mathbf{d})$. 
The field of rational GL(d)-invariants on $C$, denoted by $k(C)^{\mathrm{GL}(\mathbf{d})}$, is defined as follows:

$$
k(C)^{\mathrm{GL}(\mathbf{d})}=\{\varphi \in k(C) \mid g \varphi=\varphi, \forall g \in \mathrm{GL}(\mathbf{d})\} .
$$

Our motivation for studying such fields of rational invariants is twofold: on one hand, $k(C)^{\mathrm{GL}(\mathrm{d})}$ is the function field of the rational quotient (in the sense of Rosenlicht) of $C$ by $\mathrm{GL}(\mathbf{d})$, which parametrizes the generic $A$-modules in $C$. Hence, these fields play a key role in the generic representation theory of $A$.

On the other hand, there are important projective (parameterizing) varieties, such as the Hilbert scheme of points on $\mathbb{P}^{2}$ (see [1]) or various moduli spaces of sheaves over rational surfaces (see 38]), whose function fields can be viewed as fields of rational invariants for bound quiver algebras. Thus, it is desirable to know how methods and ideas from representation theory of algebras can be used to shed light on the rationality question for such varieties.

In what follows, we explain how to reduce the problem of describing fields of rational invariants on irreducible components of module varieties to the case where the irreducible components involved are indecomposable. But first we recall some fundamental facts from rational invariant theory for which we refer the reader to 32 and the references therein. Let $G$ be a linear algebraic group acting regularly on an irreducible variety $X$. The field $k(X)^{G}$ of $G$-invariant rational functions on $X$ is always finitely generated over $k$ since it is a subfield of $k(X)$ which is finitely generated over $k$. A rational quotient of $X$ by (the action of) $G$ is an irreducible variety $Y$ such that $k(Y)=k(X)^{G}$ together with the dominant rational map $\pi: X \rightarrow Y$ induced by the inclusion $k(X)^{G} \subset k(X)$. In [37], Rosenlicht shows that there is always a rational quotient of $X$ by $G$, which is uniquely defined up to birational isomorphism. In fact, it is proved that there is a $G$-invariant open and dense subset $X_{0}$ of $X$ such that the restriction of $\pi$ to $X_{0}$ is a dominant regular morphism and $\pi^{-1}(\pi(x))=G x$ for all $x \in X_{0}$ (see [37, Theorem 2] or [9, Section 1.6]). Furthermore, one can show that a rational quotient $\pi: X \rightarrow Y$ satisfies the following universal property (see [32, Remark 2.4 and 2.5] or [31, Section 2.4]): Let $\rho: X \rightarrow Y^{\prime}$ be a rational map such that $\rho^{-1}(\rho(x))=G x$ for $x \in X$ in general position. Then there exists a rational map

$$
\bar{\rho}: Y \rightarrow Y^{\prime}
$$

such that $\rho=\bar{\rho} \circ \pi$. If in addition $\rho$ is dominant, then $\bar{\rho}$ becomes a birational isomorphism. One usually writes $X / G$ in place of $Y$ and call it the rational quotient of $X$ by $G$.

Remark 1. From the discussion above and using the theorem on the generic fiber, one can immediately see that

$$
\text { tr. } \operatorname{deg} k(X)^{G}=\operatorname{dim} X-\operatorname{dim} G+\min \left\{\operatorname{dim} \operatorname{Stab}_{G}(x) \mid x \in X\right\} .
$$

This formula combined with Lemma 3 shows that for a tame algebra $A$, a dimension vector $\mathbf{d}$, and an indecomposable irreducible component $C \subseteq \bmod (A, \mathbf{d})$, tr. $\operatorname{deg} k(C)^{\mathrm{GL}(\mathbf{d})} \in\{0,1\}$.

In what follows, if $R$ is an integral domain, we denote its field of fractions by $\operatorname{Quot}(R)$. Moreover, if $K / k$ is a field extension and $m$ is a positive integer, we define $S^{m}(K / k)$ to be the field $\left(\operatorname{Quot}\left(K^{\otimes m}\right)\right)^{S_{m}}$, where $S_{m}$ denotes the symmetric group on $m$ letters. The latter is, in fact, the same as $\operatorname{Quot}\left(\left(K^{\otimes m}\right)^{S_{m}}\right)$ since $S_{m}$ is a finite group. 
Now we are ready to prove the following reduction result (compare to [14, Proposition 4.7]):

Proposition 4. Let $C \subseteq \bmod (A, \mathbf{d})$ be an irreducible component and let

$$
C=\overline{\bigoplus_{i=1}^{n} C_{i}^{\oplus m_{i}} \oplus \bigoplus_{j=n+1}^{l} C_{j}}
$$

be the generic decomposition of $C$ where $C_{i} \subseteq \bmod \left(\Lambda, \mathbf{d}_{i}\right), 1 \leq i \leq l$, are indecomposable irreducible components with $C_{n+1}, \ldots, C_{l}$ orbit closures, $m_{1}, \ldots, m_{n}$ are positive integers, and $\mathbf{d}_{i} \neq \mathbf{d}_{j}, \forall 1 \leq i \neq j \leq n$. Then,

$$
k(C)^{\mathrm{GL}(\mathbf{d})} \simeq \operatorname{Quot}\left(\bigotimes_{i=1}^{n} S^{m_{i}}\left(k\left(C_{i}\right)^{\mathrm{GL}\left(\mathbf{d}_{i}\right)} / k\right)\right) .
$$

Proof. Note that we can write

$$
C=\overline{C^{\prime} \oplus C^{\prime \prime}}
$$

where:

- $C^{\prime}=\overline{\bigoplus_{i=1}^{n} C_{i}^{\oplus m_{i}}}$ is an irreducible component of $\bmod \left(A, \mathbf{d}^{\prime}\right)$ with $\mathbf{d}^{\prime}=$ $\sum_{i=1}^{n} \mathbf{d}_{i}$;

- $C^{\prime \prime}=\overline{\mathrm{GL}\left(\mathbf{d}^{\prime \prime}\right) M}$ with $\mathbf{d}^{\prime \prime}=\sum_{j=n+1}^{l} \mathbf{d}_{j}$.

Now, Proposition 4.7 in 14 tells us that

$$
k\left(C^{\prime}\right)^{\mathrm{GL}\left(\mathbf{d}^{\prime}\right)} \simeq \operatorname{Quot}\left(\bigotimes _ { i = 1 } ^ { n } S ^ { m _ { i } } \left(k\left(C_{i}\right)^{\left.\left.\mathrm{GL}\left(\mathbf{d}_{i}\right) / k\right)\right) .}\right.\right.
$$

The proof will follow from the lemma below.

Lemma 5. Assume that $C \subseteq \bmod (A, \mathbf{d})$ can be decomposed as

$$
C=\overline{C^{\prime} \oplus C^{\prime \prime}},
$$

where $C^{\prime} \subseteq \bmod \left(A, \mathbf{d}^{\prime}\right)$ is a $\mathrm{GL}\left(\mathbf{d}^{\prime}\right)$-invariant irreducible closed subvariety, $C^{\prime \prime}=$ $\overline{\mathrm{GL}\left(\mathbf{d}^{\prime \prime}\right) M} \subseteq \bmod \left(A, \mathbf{d}^{\prime \prime}\right)$, and $\mathbf{d}=\mathbf{d}^{\prime}+\mathbf{d}^{\prime \prime}$. Then,

$$
k(C)^{\mathrm{GL}(\mathbf{d})} \simeq k\left(C^{\prime}\right)^{\mathrm{GL}\left(\mathbf{d}^{\prime}\right)} .
$$

Proof. Fix a decomposition $k^{\mathbf{d}(x)}=k^{\mathbf{d}^{\prime}(x)} \oplus k^{\mathbf{d}^{\prime \prime}(x)}$ for all $x \in Q_{0}$, and then embed $C^{\prime} \times C^{\prime \prime}$ diagonally into $C$, and $\mathrm{GL}\left(\mathbf{d}^{\prime}\right) \times \mathrm{GL}\left(\mathbf{d}^{\prime \prime}\right)$ diagonally into $\mathrm{GL}(\mathbf{d})$. Denote GL(d) by $G, \operatorname{GL}\left(\mathbf{d}^{\prime}\right) \times\{\mathbf{1}\}$ by $H$, and let $S=C^{\prime} \times\{M\}$. Then, it is easy to see that $S$ is an irreducible $H$-invariant closed subvariety of $C$ such that:

(1) $\overline{G S}=C$;

(2) for any $s \in S$, we have $G s \cap S=H s$.

Now, let $\pi: C \rightarrow C / G$ be the rational quotient of $C$ by $G$. Then, (1) ensures that the restriction $\rho$ of $\pi$ to $S$ is a well-defined dominant rational map, and (2) simply says that for generic $s \in S, \rho^{-1}(\rho(s))=H s$. It follows from the universal property for rational quotients that $\rho$ is the rational quotient of $S$ by $H$, and so $k(C)^{\mathrm{GL}(\mathbf{d})} \simeq k(S)^{H}=k\left(C^{\prime}\right)^{\mathrm{GL}\left(\mathbf{d}^{\prime}\right)}$. 
Remark 2. This lemma tells us that, for the purposes of computing rational invariants, we can always get rid of the orbit closures that occur in a generic decomposition. If, additionally, the other irreducible components that occur in a generic decomposition can be separated as in Proposition 4 then we have a further reduction in the fields of rational invariants.

\section{Moduli spaces of modules}

Let $A=k Q / I$ be a bound quiver algebra and let $\mathbf{d} \in \mathbb{Z}_{\geq 0}^{Q_{0}}$ be a dimension vector of $A$. The ring of invariants $\mathrm{I}(A, \mathbf{d}):=k[\bmod (A, \mathbf{d})]^{\mathrm{GL}(\mathbf{d})}$ turns out to be precisely the base field $k$ since $A$ is finite-dimensional. However, the action of the subgroup $\mathrm{SL}(\mathbf{d}) \subseteq \mathrm{GL}(\mathbf{d})$, defined by

$$
\mathrm{SL}(\mathbf{d})=\prod_{x \in Q_{0}} \mathrm{SL}(\mathbf{d}(x), k),
$$

provides us with a highly non-trivial ring of semi-invariants. Note that any $\theta \in \mathbb{Z}^{Q_{0}}$ defines a rational character $\chi_{\theta}: \mathrm{GL}(\mathbf{d}) \rightarrow k^{*}$ by

$$
\chi_{\theta}\left((g(x))_{x \in Q_{0}}\right)=\prod_{x \in Q_{0}}(\operatorname{det} g(x))^{\theta(x)} .
$$

In this way, we can identify $\Gamma=\mathbb{Z}^{Q_{0}}$ with the group $X^{\star}(\mathrm{GL}(\mathbf{d}))$ of rational characters of $\mathrm{GL}(\mathbf{d})$, assuming that $\mathbf{d}$ is a sincere dimension vector. In general, we have only the natural epimorphism $\Gamma \rightarrow X^{*}(\mathrm{GL}(\mathbf{d}))$. We also refer to the rational characters of $\mathrm{GL}(\mathbf{d})$ as (integral) weights of $A$ (or $Q)$ ).

Let us now consider the ring of semi-invariants $\operatorname{SI}(A, \mathbf{d}):=k[\bmod (A, \mathbf{d})]^{\mathrm{SL}(\mathbf{d})}$. As $\mathrm{SL}(\mathbf{d})$ is the commutator subgroup of $\mathrm{GL}(\mathbf{d})$ and $\mathrm{GL}(\mathbf{d})$ is linearly reductive, we have

$$
\mathrm{SI}(A, \mathbf{d})=\bigoplus_{\theta \in X^{\star}(\mathrm{GL}(\mathbf{d}))} \mathrm{SI}(A, \mathbf{d})_{\theta}
$$

where

$$
\mathrm{SI}(A, \mathbf{d})_{\theta}=\{f \in k[\bmod (A, \mathbf{d})] \mid g f=\theta(g) f \text { for all } g \in \mathrm{GL}(\mathbf{d})\}
$$

is called the space of semi-invariants on $\bmod (A, \mathbf{d})$ of weight $\theta$. For an irreducible component $C \subseteq \bmod (A, \mathbf{d})$, we similarly define the ring of semi-invariants $\operatorname{SI}(C):=$ $k[C]^{\mathrm{SL}(\mathbf{d})}$, and the space $\mathrm{SI}(C)_{\theta}$ of semi-invariants on $C$ of weight $\theta \in \mathbb{Z}^{Q_{0}}$.

Following King [28, an $A$-module $M$ is said to be $\theta$-semi-stable if $\theta(\operatorname{dim} M)=0$ and $\theta\left(\operatorname{dim} M^{\prime}\right) \leq 0$ for all submodules $M^{\prime} \leq M$. We say that $M$ is $\theta$-stable if $M$ is non-zero, $\theta(\operatorname{dim} M)=0$, and $\theta\left(\operatorname{dim} M^{\prime}\right)<0$ for all submodules $\{0\} \neq M^{\prime}<M$. We denote by $\bmod (A)_{\theta}^{s s}$ the full subcategory of $\bmod (A)$ consisting of the $\theta$-semistable modules. It is easy to see that $\bmod (A)_{\theta}^{s s}$ is a full exact abelian subcategory of $\bmod (A)$ which is closed under extensions and whose simple objects are precisely the $\theta$-stable modules. Moreover, $\bmod (A)_{\theta}^{s s}$ is Artinian and Noetherian, and hence every $\theta$-semi-stable $A$-module has a Jordan-Hölder filtration in $\bmod (A)_{\theta}^{s s}$.

Now, let us consider the (possibly empty) open subsets

$$
\bmod (A, \mathbf{d})_{\theta}^{s s}=\{M \in \bmod (A, \mathbf{d}) \mid M \text { is } \theta \text {-semi-stable }\}
$$

and

$$
\bmod (A, \mathbf{d})_{\theta}^{s}=\{M \in \bmod (A, \mathbf{d}) \mid M \text { is } \theta \text {-stable }\}
$$


of d-dimensional $\theta$ (-semi)-stable $A$-modules. Using methods from Geometric Invariant Theory, King showed in 28 that the projective variety

$$
\mathcal{M}(A, \mathbf{d})_{\theta}^{s s}:=\operatorname{Proj}\left(\bigoplus_{n \geq 0} \operatorname{SI}(A, \mathbf{d})_{n \theta}\right)
$$

is a GIT-quotient of $\bmod (A, \mathbf{d})_{\theta}^{s s}$ by the action of $\operatorname{PGL}(\mathbf{d})$, where $\operatorname{PGL}(\mathbf{d})=$ $\mathrm{GL}(\mathbf{d}) / T_{1}$ and $T_{1}=\left\{\left(\lambda \operatorname{Id}_{\mathbf{d}(x)}\right)_{x \in Q_{0}} \mid \lambda \in k^{*}\right\} \leq \mathrm{GL}(\mathbf{d})$. Moreover, there is a (possibly empty) open subset $\mathcal{M}(A, \mathbf{d})_{\theta}^{s}$ of $\mathcal{M}(A, \mathbf{d})_{\theta}^{s s}$ which is a geometric quotient of $\bmod (A, \mathbf{d})_{\theta}^{s}$ by $\mathrm{PGL}(\mathbf{d})$. We say that $\mathbf{d}$ is a $\theta$-(semi-)stable dimension vector if $\bmod (A, \mathbf{d})_{\theta}^{(s) s} \neq \emptyset$.

For an irreducible component $C \subseteq \bmod (A, \mathbf{d})$, we similarly define $C_{\theta}^{s s}, C_{\theta}^{s}$, $\mathcal{M}(C)_{\theta}^{s s}$, and $\mathcal{M}(C)_{\theta}^{s}$. One then has that the points of $\mathcal{M}(C)_{\theta}^{s}$ correspond bijectively to the isomorphism classes of $\theta$-stable modules in $C$. We say that $C$ is $\theta$-(semi-) stable if $C_{\theta}^{(s) s} \neq \emptyset$.

Remark 3. Let $C \subseteq \bmod (A, \mathbf{d})$ be an irreducible component and let $\theta \in \mathbb{Z}^{Q_{0}}$ be so that $C_{\theta}^{s} \neq \emptyset$. Let $\pi: C \rightarrow \mathcal{M}(C)_{\theta}^{s}$ be the dominant rational morphism which is represented by the geometric quotient morphism $C_{\theta}^{s} \rightarrow \mathcal{M}(C)_{\theta}^{s}$. Since for any $M \in C_{\theta}^{s}, \pi^{-1}(\pi(M))=\mathrm{GL}(\mathbf{d}) M$, we conclude that $\mathcal{M}(C)_{\theta}^{s}$ is the rational quotient of $C$ by $\mathrm{GL}(\mathbf{d})$. In particular, we have that $k(C)^{\mathrm{GL}(\mathbf{d})} \simeq k\left(\mathcal{M}(C)_{\theta}^{s s}\right)$.

A $\theta$-semi-stable irreducible component $C \subseteq \bmod (A, \mathbf{d})$ is said to be $\theta$-wellbehaved if, whenever $\mathbf{d}^{\prime}$ is the dimension vector of a factor of a Jordan-Hölder filtration in $\bmod (A)_{\theta}^{s s}$ of a generic $A$-module in $C$ and $C_{1}, C_{2} \subseteq \bmod \left(A, \mathbf{d}^{\prime}\right)$ are two distinct irreducible components, $C_{1, \theta}^{s} \cap C_{2, \theta}^{s}=\emptyset$. (We should point out that this notion is slightly more general than the one in [15, Section 3.3].)

Remark 4. It follows from the work of Bobiński and Skowroński in [6] that for a tame quasi-tilted algebra, any $\theta$-semi-stable irreducible component is $\theta$-well-behaved.

We will prove in Section 5 that the same is true for triangular gentle algebras.

Let $C$ be a $\theta$-well-behaved irreducible component of $\bmod (A, \mathbf{d})$. We say that

$$
C=C_{1} \dot{+} \ldots \dot{+} C_{l}
$$

is the $\theta$-stable decomposition of $C$ if:

- the $C_{i} \subseteq \bmod \left(A, \mathbf{d}_{i}\right), 1 \leq i \leq l$, are $\theta$-stable irreducible components;

- the generic $A$-module $M$ in $C$ has a finite filtration $0=M_{0} \subseteq M_{1} \subseteq \cdots \subseteq$ $M_{l}=M$ of submodules such that there is a permutation $\sigma \in S_{l}$ such that $M_{j} / M_{j-1}$ is isomorphic to a $\theta$-stable module in $C_{\sigma(j)}$ for every $1 \leq j \leq l$.

We call $C_{1}, \ldots, C_{l}$ the $\theta$-stable summands of $C$. To prove the existence and uniqueness of the $\theta$-stable decomposition of $C$, first note that the irreducible variety $C_{\theta}^{s s}$ is a disjoint union of sets of the form $\mathcal{F}_{\left(C_{i}\right)_{1<i<l}}$, where each $\mathcal{F}_{\left(C_{i}\right)_{1<i<l}}$ consists of those modules $M \in C$ that have a finite filtration $0=M_{0} \subseteq M_{1} \subseteq \cdots \subseteq M_{l}=M$ of submodules with each factor $M_{j} / M_{j-1}$ isomorphic to a $\theta$-stable module in one of the $C_{i}, 1 \leq i \leq l$. (Note that the $\theta$-well-behavedness of $C$ is used to ensure that the union above is indeed disjoint.) Next, it is not difficult to show that each $\mathcal{F}_{\left(C_{i}\right)_{1 \leq i \leq l}}$ is constructible (see for example [17, Section 3]). Hence, there is a unique (up to permutation) sequence $\left(C_{i}\right)_{1 \leq i \leq l}$ of $\theta$-stable irreducible components for which $\mathcal{F}_{\left(C_{i}\right)_{1 \leq i \leq l}}$ contains an open and dense subset of $C_{\theta}^{s s}$ (or $C$ ). 
Now, we are ready to state the following reduction result from [15, Theorem 1.4]:

Theorem 6. Let $A=k Q / I$ be a bound quiver algebra and let $C \subseteq \bmod (A, \mathbf{d})$ be a $\theta$-well-behaved irreducible component where $\theta$ is an integral weight of $A$. Let

$$
C=m_{1} \cdot C_{1} \dot{+} \ldots \dot{+} m_{n} \cdot C_{n}
$$

be the $\theta$-stable decomposition of $C$ where $C_{i} \subseteq \bmod \left(A, \mathbf{d}_{i}\right), 1 \leq i \leq n$, are $\theta$-stable irreducible components and $\mathbf{d}_{i} \neq \mathbf{d}_{j}$ for all $1 \leq i \neq j \leq n$. Assume that $C$ is a

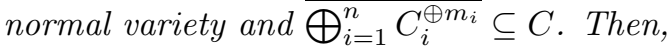

$$
\mathcal{M}(C)_{\theta}^{s s} \cong S^{m_{1}}\left(\mathcal{M}\left(C_{1}\right)_{\theta}^{s s}\right) \times \ldots \times S^{m_{n}}\left(\mathcal{M}\left(C_{n}\right)_{\theta}^{s s}\right) .
$$

Note that this reduction result allows us to "break" a moduli space of modules into smaller ones. We show next that these smaller moduli spaces are rather wellbehaved, at least in the tame case.

Proposition 7. Let $A=k Q / I$ be a tame bound quiver algebra, $\mathbf{d}$ a generic root of $A$, and $C \subseteq \bmod (A, \mathbf{d})$ an indecomposable irreducible component. Then, the following statements hold:

(1) For any weight $\theta \in \mathbb{Z}^{Q_{0}}$ with $C_{\theta}^{s s} \neq \emptyset, \mathcal{M}(C)_{\theta}^{s s}$ is either a point or projective curve.

(2) If $\theta \in \mathbb{Z}^{Q_{0}}$ is so that $C_{\theta}^{s} \neq \emptyset$, then $\mathcal{M}(C)_{\theta}^{s s}$ is rational. If, in addition, $C$ is normal, then $\mathcal{M}(C)_{\theta}^{s s}$ is either a point or $\mathbb{P}^{1}$.

(3) If $C$ is a Schur component, then $k(C)^{\mathrm{GL}(\mathbf{d})}$ is a rational field of transcendence degree at most one.

Proof. If $C$ is an obit closure, then (1) - (3) are obviously true. Otherwise, we have seen in Section 2.2 that

$$
C=\overline{\bigcup_{\lambda \in \mathcal{U}} \mathrm{GL}(\mathbf{d}) f(\lambda)}
$$

where $\left(\mathcal{U} \subseteq k^{*}, f: \mathcal{U} \rightarrow C\right)$ is a parameterizing pair.

(1) Let $\theta \in \mathbb{Z}^{Q_{0}}$ be an integral weight and $\pi: C_{\theta}^{s s} \rightarrow \mathcal{M}(C)_{\theta}^{s s}$ be the quotient morphism. Then, for a generic module $M_{0} \in C_{\theta}^{s s}$, we have

$$
\operatorname{dim} C-\operatorname{dim} \mathcal{M}(C)_{\theta}^{s s}=\operatorname{dim} \pi^{-1}\left(\pi\left(M_{0}\right)\right) \geq \operatorname{dim} \overline{\mathrm{GL}(\mathbf{d}) M_{0}} .
$$

So, $\operatorname{dim} \mathcal{M}(C)_{\theta}^{s s} \leq \operatorname{dim} C-\operatorname{dim} \operatorname{GL}(\mathbf{d})+\min \left\{\operatorname{dim}_{k} \operatorname{End}_{A}(M) \mid M \in C\right\}$, which is at most 1 by Lemma 3 . This proves (1).

(2) Choose a non-empty open subset $X_{0} \subseteq C$ such that $X_{0} \subseteq \bigcup_{\lambda \in \mathcal{U}} \mathrm{GL}(\mathbf{d}) f(\lambda) \cap$ $C_{\theta}^{s}$. We can certainly assume that $X_{0}$ is $\mathrm{GL}(\mathbf{d})$-invariant since otherwise we can simply work with $\bigcup_{g \in \mathrm{GL}(\mathbf{d})} g X_{0}$. It is then easy to see that $\mathcal{U}_{0}:=\{\lambda \in \mathcal{U} \mid f(\lambda) \in$ $\left.X_{0}\right\}$ is a non-empty open subset of $\mathcal{U}$.

Now, let $\varphi: \mathcal{U}_{0} \rightarrow \mathcal{M}(C)_{\theta}^{s s}$ be the morphism defined by $\varphi(\lambda)=\pi(f(\lambda)), \forall \lambda \in \mathcal{U}_{0}$. Notice that $\varphi$ is injective. Indeed, let $\lambda_{1}, \lambda_{2} \in \mathcal{U}_{0}$ be so that $\varphi\left(\lambda_{1}\right)=\varphi\left(\lambda_{2}\right)$. Then, $\pi\left(f\left(\lambda_{1}\right)\right)=\pi\left(f\left(\lambda_{2}\right)\right)$, which is equivalent to $\overline{\operatorname{GL}(\mathbf{d}) f\left(\lambda_{1}\right)} \cap \overline{\operatorname{GL}(\mathbf{d}) f\left(\lambda_{2}\right)} \cap C_{\theta}^{s s} \neq \emptyset$. Since $f\left(\lambda_{1}\right)$ and $f\left(\lambda_{2}\right)$ are both $\theta$-stable, we know that their orbits are closed in $C_{\theta}^{s s}$. It is now clear that $f\left(\lambda_{1}\right) \simeq f\left(\lambda_{2}\right)$, which implies that $\lambda_{1}=\lambda_{2}$.

The injectivity of $\varphi$ together with the fact that $\operatorname{dim} \mathcal{U}_{0}=1$ and $\operatorname{dim} \mathcal{M}(C)_{\theta}^{s s} \leq 1$ implies that $\varphi$ is an injective dominant morphism, and hence is birational. This shows that $\mathcal{M}(C)_{\theta}^{s s}$ is a rational projective curve. 
If, in addition, $C$ is normal, then so is any good quotient of $C$ (see for example [25, Proposition 2.15]). In particular, under this extra assumption, $\mathcal{M}(C)_{\theta}^{s s}$ is a rational normal projective curve, i.e. $\mathcal{M}(C)_{\theta}^{s s}$ is precisely $\mathbb{P}^{1}$.

(3) Notice that $\left\{\lambda \in \mathcal{U} \mid \operatorname{dim}_{k} \operatorname{End}_{A}(f(\lambda))=\min \left\{\operatorname{dim}_{k} \operatorname{End}_{A}(M) \mid M \in C\right\}\right\}$ is a non-empty open subset of $\mathcal{U}$. From this we deduce that $C$ contains infinitely many, pairwise non-isomorphic, Schur $A$-modules. Hence, $C$ must contain a homogenous Schur $A$-module $M$ by [18, Theorem D]. It has been proved in [16, Lemma 11] that $M$ is $\theta^{M}$-stable where $\theta^{M}$ is a specific integral weight associated to $M$ (see also Section 6). Using Remark 3 and part (2), we can now see that

$$
k(C)^{\mathrm{GL}(\mathbf{d})} \simeq k\left(\mathcal{M}(C)_{\theta^{M}}^{s s}\right) \simeq k(t) .
$$

Remark 5. As a consequence of the proposition above and Theorem 6 , we obtain that for a tame algebra $A$ and an irreducible component $C \subseteq \bmod (A, \mathbf{d})$ for which Theorem 6 is applicable, the moduli space $\mathcal{M}(C)_{\theta}^{s s}$ is a rational variety.

\section{ACyClic Gentle Algebras}

Gentle algebras are a particularly well-behaved class of string algebras that have recently enjoyed a resurgence in popularity, primarily due to their appearance in the study of cluster algebras arising from unpunctured surfaces (see [2]). String algebras are (non-hereditary) tame algebras whose indecomposable modules can be parameterized by certain walks on the underlying quiver $Q$ not passing through the ideal $I$, and whose irreducible morphisms can be described by operations on these walks [10. The invariant theory for a particular class of triangular gentle algebras was first studied in detail by Kraśkiewicz and Weyman [30], and then by the second author in [11 13. In these latter works, the irreducible components of triangular gentle algebras are determined. Their rings of semi-invariants are shown to be semigroup rings, and the generic modules in irreducible components are constructed. This allows for the calculation of the quotients $\mathcal{M}(C)_{\theta}^{s s}$ for some special choices of $C$ and $\theta$ in [12] and for more general choices of $C$ in this article.

A bound quiver algebra $k Q / I$ is called a gentle algebra if the following properties hold:

(1) for each vertex $x \in Q_{0}$ there are at most two arrows with head $x$, and at most two arrows with tail $x$;

(2) for any arrow $b \in Q_{1}$, there is at most one arrow $a \in Q_{1}$ and at most one arrow $c \in Q_{1}$ such that $a b \notin I$ and $b c \notin I$;

(3) for each arrow $b \in Q_{1}$ there is at most one arrow $a \in Q_{1}$ with $t a=h b$ (resp. at most one arrow $c \in Q_{1}$ with $\left.h c=t b\right)$ such that $a b \in I$ (resp. $\left.b c \in I\right)$;

(4) $I$ is generated by paths of length 2 .

The bound quiver algebra $k Q / I$ is called a gentle algebra.

In [13], colorings of a quiver were introduced to understand the module varieties of triangular gentle algebras. A coloring $c$ of a quiver $Q$ is a surjective map $c$ : $Q_{1} \rightarrow S$, where $S$ is some finite set whose elements we call colors, such that $c^{-1}(s)$ is a directed path for all $s \in S$. Given a coloring $c$ of $Q$, define the coloring ideal $I_{c}$ to be the two-sided ideal in $k Q$ generated by monochromatic paths of length two, i.e. $I_{c}=\langle b a| c(b)=c(a)$ and $\left.h a=t b\right\rangle$. 
Proposition 8 ([13, Proposition 2.6]). If $k Q / I$ is a triangular gentle algebra, then there is a coloring c of $Q$ such that $I=I_{c}$.

Let $c$ be a coloring of $Q, A=k Q / I_{c}$, and $\mathbf{d}$ a dimension vector of $A$. The irreducible components of $\bmod (A, \mathbf{d})$ can be parameterized by introducing the concept of a rank function. A rank function (with respect to $\mathbf{d}$ ) is a map $\mathbf{r}: Q_{1} \rightarrow \mathbb{N}$ satisfying the inequalities

$$
\mathbf{r}(a)+\mathbf{r}(b) \leq \mathbf{d}(x)
$$

whenever $h a=t b=x$ and $c(a)=c(b)$ (with the degenerate inequalities $\mathbf{r}(a) \leq \mathbf{d}(x)$ or $\mathbf{r}(b) \leq \mathbf{d}(x)$ when either $b$ or $a$ fails to exist). The rank function $\mathbf{r}$ is called maximal if it is so under the coordinate-wise partial order (namely $\mathbf{r}^{\prime} \leq \mathbf{r}$ if and only if $\mathbf{r}^{\prime}(a) \leq \mathbf{r}(a)$ for all $\left.a \in Q_{1}\right)$. Note that a rank function $\mathbf{r}$ defines a closed subvariety of $\bmod (A, \mathbf{d})$ :

$$
\bmod (A, \mathbf{d}, \mathbf{r}):=\left\{V \in \bmod (A, \mathbf{d}) \mid \operatorname{rank} V(a) \leq \mathbf{r}(a), \forall a \in Q_{1}\right\} .
$$

It was shown in 13 that every irreducible component of $\bmod (A, \mathbf{d})$ is of the form $\bmod (A, \mathbf{d}, \mathbf{r})$ for $\mathbf{r}$ maximal. These varieties can then be viewed as products of varieties of complexes taken along each colored path. DeConcini-Strickland [19] showed that varieties of complexes are normal, and thus $\bmod (A, \mathbf{d}, \mathbf{r})$ is normal for any choice of $\mathbf{d}, \mathbf{r}$.

5.1. Up and down graphs. We now focus exclusively on the case in which $A=$ $k Q / I_{c}$ is a triangular gentle algebra. Under this restriction, each vertex has at most two colors incident to it. Denote by $X$ the set of pairs $(x, s) \in Q_{0} \times S$ such that $s$ is the color of an arrow incident to $x$. A sign function is a map $\epsilon: \mathcal{X} \rightarrow\{ \pm 1\}$ satisfying the property that $\epsilon(x, s)=-\epsilon\left(x, s^{\prime}\right)$ if $(x, s),\left(x, s^{\prime}\right)$ are distinct elements of $x$.

The up and down graph $\Gamma(Q, c, \mathbf{d}, \mathbf{r}, \epsilon)$ is the directed graph with vertices $\left\{v_{j}^{x} \mid\right.$ $\left.x \in Q_{0}, j=1, \ldots, \mathbf{d}(x)\right\}$ and arrows $\left\{f_{j}^{a} \mid a \in Q_{1}, j=1, \ldots, \mathbf{r}(a)\right\}$ so that

$$
\begin{aligned}
& t f_{j}^{a}= \begin{cases}v_{j}^{t a} & \text { if } \epsilon(t a, c(a))=1, \\
v_{\mathbf{d}(t a)-j+1}^{t a} & \text { if } \epsilon(t a, c(a))=-1,\end{cases} \\
& h f_{j}^{a}= \begin{cases}v_{\mathbf{d}(h a)-j+1}^{h a} & \text { if } \epsilon(h a, c(a))=1, \\
v_{j}^{h a} & \text { if } \epsilon(h a, c(a))=-1 .\end{cases}
\end{aligned}
$$

There is an obvious morphism of quivers $\pi: \Gamma(Q, c, \mathbf{d}, \mathbf{r}, \epsilon) \rightarrow Q$ with $\pi\left(v_{j}^{x}\right)=x$ and $\pi\left(f_{j}^{a}\right)=a$. This, in turn, gives rise to a pushforward map $\pi_{*}: \operatorname{rep}(\Gamma(Q, c, \mathbf{d}, \mathbf{r}, \epsilon))$ $\rightarrow \operatorname{rep}(Q)$, where if $V=\left(V\left(v_{j}^{x}\right), V\left(f_{j}^{a}\right)\right) \in \operatorname{rep}(\Gamma(Q, c, \mathbf{d}, \mathbf{r}, \epsilon))$, then

$$
\begin{aligned}
& \pi_{*}(V)(x):=\bigoplus_{j=1, \ldots, \mathbf{d}(x)} V\left(v_{j}^{x}\right), \\
& \pi_{*}(V)(a):=\sum_{j=1, \ldots, \mathbf{r}(a)} V\left(f_{j}^{a}\right) .
\end{aligned}
$$

Notice that since $\mathbf{r}$ is a rank function, by construction of $\Gamma(Q, c, \mathbf{d}, \mathbf{r}, \epsilon)$ we have that $\pi_{*}(V)(b) \circ \pi_{*}(V)(a)=0$ whenever $h a=t b$ and $c(a)=c(b)$. Therefore the image of $\pi_{*}$ lies in $\bmod (A)$. Furthermore, each vertex in $\Gamma$ is incident to at most two arrows, and so the connected components of $\Gamma(Q, c, \mathbf{d}, \mathbf{r}, \epsilon)$ are either chains or cycles (components which we refer to as strings and bands, respectively). Let $B$ be the set of band components in $\Gamma$, and for $b \in B$ choose a sink $\Theta(b)$ contained in $b$. 
For each $\underline{\lambda}=\left(\lambda_{b}\right)_{b \in B} \in k^{B}$, the representation $\widetilde{M}(Q, c, \mathbf{d}, \mathbf{r}, \epsilon, \underline{\lambda})$ of $\Gamma(Q, c, \mathbf{d}, \mathbf{r}, \epsilon)$ is defined to be the representation with $\widetilde{M}\left(v_{j}^{x}\right)=k$ for all $v_{j}^{x} \in \Gamma(Q, c, \mathbf{d}, \mathbf{r}, \epsilon)_{0}$ with the action of the arrows given by multiplication as follows:

$$
\widetilde{M}\left(f_{j}^{a}\right)= \begin{cases}\lambda_{b} & \text { if } h f_{j}^{a}=\Theta(b) \text { and } \epsilon(h a, c(a))=-1 \\ 1 & \text { otherwise. }\end{cases}
$$

The up and down module $M(Q, c, \mathbf{d}, \mathbf{r}, \epsilon, \underline{\lambda})$ is defined to be $\pi_{*}(\widetilde{M}(Q, c, \mathbf{d}, \mathbf{r}, \epsilon, \underline{\lambda}))$. When the quiver $Q$ and coloring $c$ are understood from context, we will simply write $\Gamma(\mathbf{d}, \mathbf{r}, \epsilon)$ and $M(\mathbf{d}, \mathbf{r}, \epsilon, \underline{\lambda})$. When $\Gamma(\mathbf{d}, \mathbf{r}, \epsilon)$ consists of a single string, we call $\widetilde{M}(Q, c, \mathbf{d}, \mathbf{r}, \epsilon, \underline{\lambda})$ a string module, while if $\Gamma(\mathbf{d}, \mathbf{r}, \epsilon)$ consists of a collection of band components, then $\widetilde{M}(Q, c, \mathbf{d}, \mathbf{r}, \epsilon, \underline{\lambda})$ is called a band module when $\underline{\lambda} \in\left(k^{*}\right)^{B}$.

Example 1. Consider the following bound quiver $\left(Q, I_{c}\right)$ with the coloring as indicated by the type of arrow:

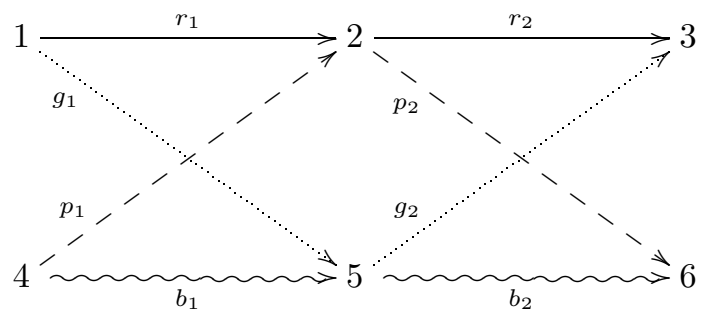

We consider the dimension vector $\mathbf{d}$ and rank function $\mathbf{r}$ indicated in the diagram below, with dimensions in boxes and ranks as decorations of the arrows:

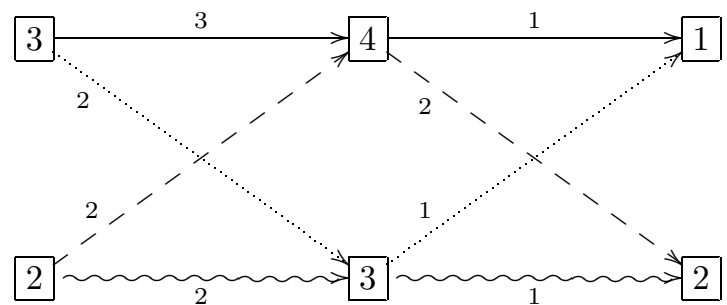

Take $\epsilon$ as indicated, where we place $\epsilon(x, c(a))$ on the arrow $a$ near the vertex $x$ :

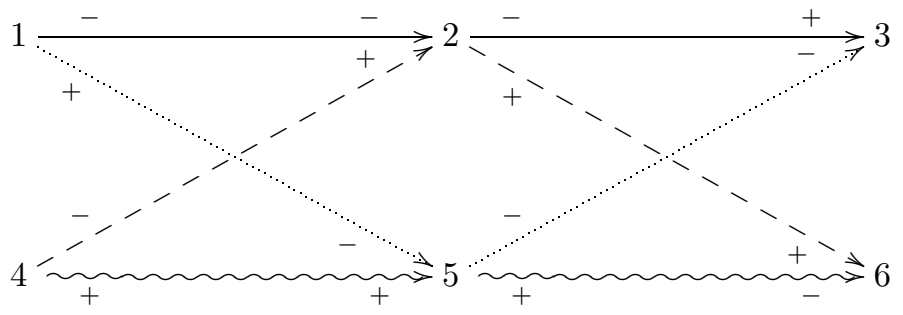

The up and down graph $\Gamma(\mathbf{d}, \mathbf{r}, \epsilon)$ consists of one band component and one string component. One possible choice of $\Theta(b)$ for the unique band is the vertex framed 
by a circle here:

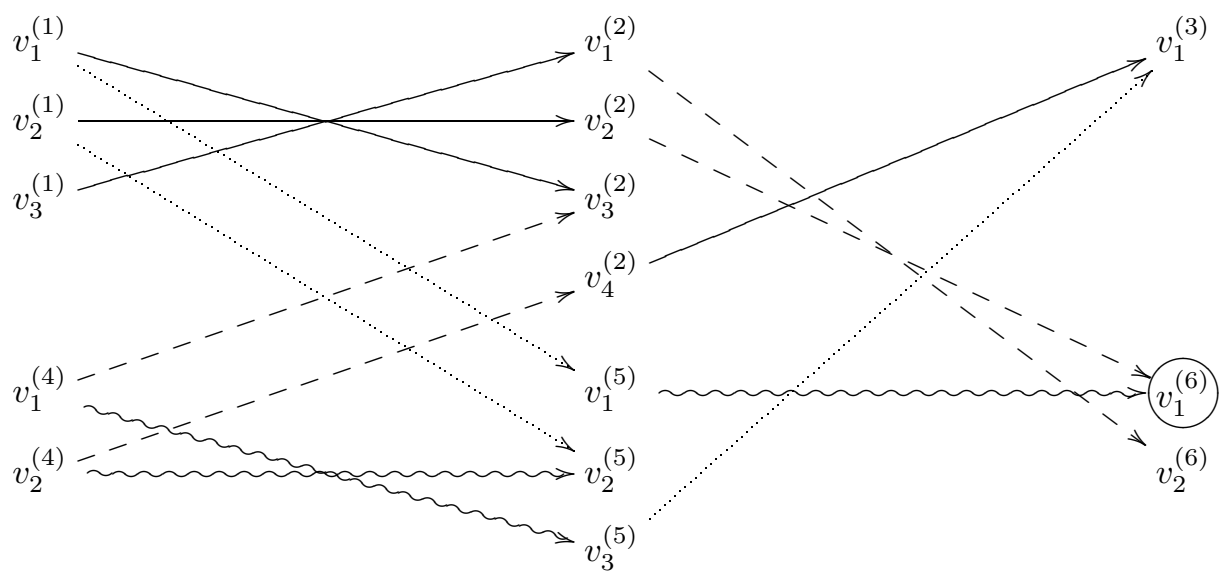

Then $M(\mathbf{d}, \mathbf{r}, \epsilon, \lambda)$ is the module given by:

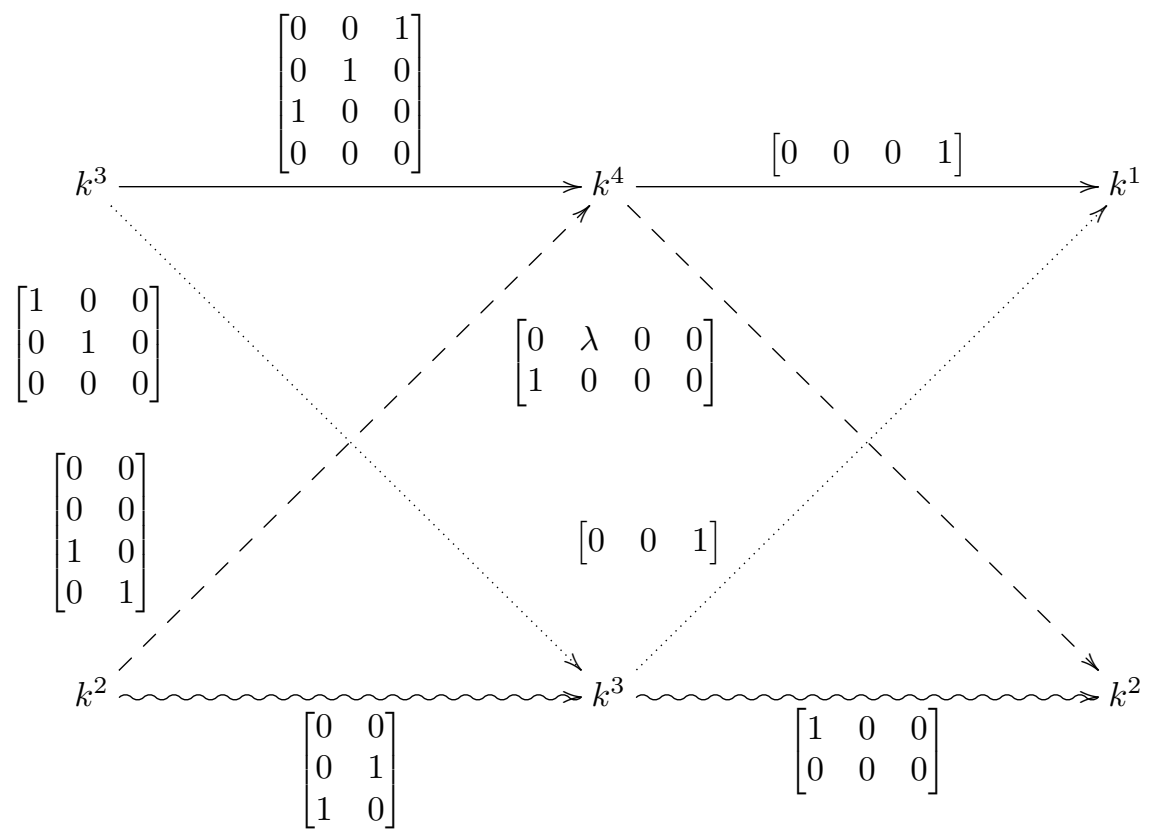

In [11, 12, an explicit minimal projective resolution for each $M(\mathbf{d}, \mathbf{r}, \epsilon, \underline{\lambda})$ is constructed and employed to show

$$
\operatorname{Ext}_{A}^{1}\left(M(\mathbf{d}, \mathbf{r}, \epsilon, \underline{\lambda}), M\left(\mathbf{d}, \mathbf{r}, \epsilon, \underline{\lambda^{\prime}}\right)\right)=0
$$

whenever $\underline{\lambda}, \underline{\lambda^{\prime}} \in\left(k^{*}\right)^{B}$ are vectors that share no common coordinates, and that when $\Gamma(\mathbf{d}, \mathbf{r}, \epsilon)$ consists of a single band component,

$$
\operatorname{Ext}_{A}^{1}(M(\mathbf{d}, \mathbf{r}, \epsilon, \underline{\lambda}), M(\mathbf{d}, \mathbf{r}, \epsilon, \underline{\lambda}))=k .
$$


In particular, these results can be exploited to show that

$$
\bmod (A, \mathbf{d}, \mathbf{r})=\overline{\bigcup_{\underline{\lambda} \in\left(k^{*}\right)^{B}} \operatorname{GL}(\mathbf{d}) M(\mathbf{d}, \mathbf{r}, \epsilon, \underline{\lambda}) .}
$$

When there are no band modules, $\bmod (A, \mathbf{d}, \mathbf{r})$ is simply the orbit closure of the rigid module $M(\mathbf{d}, \mathbf{r}, \epsilon)$. This means that in order to apply the results of Section 3 , we need only understand those irreducible components whose generic modules are direct sums of band modules.

Given these results, we notice that the family $\operatorname{GL}(\mathbf{d}) M(\mathbf{d}, \mathbf{r}, \epsilon, \underline{\lambda})$ is independent of the choice of $\epsilon$. In the subsequent sections we will suppress this variable when referring to both the up-and-down graph and the up-and-down modules.

It is important to note that the indecomposable direct summands of $M(\mathbf{d}, \mathbf{r}, \epsilon, \underline{\lambda})$ correspond to the connected components of $\Gamma(\mathbf{d}, \mathbf{r}, \epsilon)$. Thus, the graph $\Gamma(\mathbf{d}, \mathbf{r}, \epsilon)$ also gives the generic decomposition of $\bmod (A, \mathbf{d}, \mathbf{r})$, i.e., the dimension vectors $\mathbf{d}_{i}$ and rank functions $\mathbf{r}_{i}$ such that

$$
\bmod (A, \mathbf{d}, \mathbf{r})=\overline{\bmod \left(A, \mathbf{d}_{1}, \mathbf{r}_{1}\right) \oplus \ldots \oplus \bmod \left(A, \mathbf{d}_{n}, \mathbf{r}_{n}\right)},
$$

where $\bmod \left(A, \mathbf{d}_{i}, \mathbf{r}_{i}\right)$ are indecomposable irreducible components. In particular, in order to apply the reduction techniques from Proposition 4 and Theorem 6, it needs to be shown that if $\bmod \left(A, \mathbf{d}_{i}, \mathbf{r}_{i}\right)$ and $\bmod \left(A, \mathbf{d}_{j}, \mathbf{r}_{j}\right)$ are direct summands that are not orbit closures, and $\mathbf{d}_{i}=\mathbf{d}_{j}$, then $\mathbf{r}_{i}=\mathbf{r}_{j}$.

5.2. Regular irreducible components. An irreducible component $\bmod (A, \mathbf{d}, \mathbf{r})$ is called regular if the generic module $M(\mathbf{d}, \mathbf{r}, \underline{\lambda})$ is a direct sum of bands. That is, the only connected components of $\Gamma(\mathbf{d}, \mathbf{r})$ are cycles. We can characterize the ranks for which this is the case in the following proposition.

Proposition 9 ([12, Chapter 5]). The irreducible component $\bmod (A, \mathbf{d}, \mathbf{r})$ is regular if and only if the following two conditions hold:

I. for any pair of consecutive monochromatic arrows $a, b$ with $h b=t a=x$, $\mathbf{r}(a)+\mathbf{r}(b)=\mathbf{d}(x)$ (together with the degenerate conditions when either $b$ is the last arrow or a is the first arrow of a given color, in which case the other summand is suppressed);

II. for any vertex $x$ incident to only one color, $\mathbf{d}(x)=0$.

There are a number of important consequences that follow from this combinatorial requirement.

Corollary 10. If $\bmod (A, \mathbf{d})$ contains an irreducible regular component, then it is unique. Furthermore, in this case $\langle\langle\mathbf{d}, \mathbf{d}\rangle\rangle=0$.

Proof. Suppose that $\bmod (A, \mathbf{d})$ contains an irreducible regular component. We will show that any rank function $\mathbf{r}$ such that $\bmod (A, \mathbf{d}, \mathbf{r})$ is regular is determined uniquely by the dimension vector and the conditions of Proposition 9. Given any color $s \in S$, let $a_{n(s)} \cdot \ldots \cdot a_{1}$ be the full path of the elements of $c^{-1}(s)$. Let $x_{j}=h a_{j}$ and $x_{0}=t a_{1}$. Since the connected components of $\Gamma(Q, \mathbf{d}, \mathbf{r}, \epsilon)$ must be cycles, with no two colored arrows incident to the same vertex, $v_{k}^{x_{j}}$ must be incident to an arrow of color $s$ for $j=0, \ldots, n(s)$ and $k=1, \ldots, \mathbf{d}\left(x_{j}\right)$. In particular, $\mathbf{r}\left(a_{1}\right)=\mathbf{d}\left(x_{0}\right)$, and $\mathbf{r}\left(a_{j+1}\right)=\mathbf{d}\left(x_{j}\right)-\mathbf{r}\left(a_{j}\right)$, so the rank function $\mathbf{r}$ for which $\bmod (A, \mathbf{d}, \mathbf{r})$ is regular is uniquely determined by $\mathbf{d}$. As for the Euler form, one simply expresses the matrix of the form as a sum of contributions along each color, and then uses 
the fact that if $\bmod (A, \mathbf{d}, \mathbf{r})$ is regular, then for any fully colored path as above, $\sum_{j=0}^{n(s)}(-1)^{j} \mathbf{d}\left(x_{j}\right)=0$. Precise details can be found in [12, Chapter 5.1].

It will be convenient to describe the projective resolution for $X_{\underline{\lambda}}:=M(\mathbf{d}, \mathbf{r}, \underline{\lambda})$ when $\bmod (A, \mathbf{d}, \mathbf{r})$ is a regular irreducible component (the general case is handled in [11, Section 3]). This will allow us to derive a closed formula for the Schofield semi-invariants of weight $\langle\langle\mathbf{d},-\rangle\rangle$. Let us denote by $S^{0}$ and $S^{1}$ the set of sources and sinks of $\Gamma=\Gamma(\mathbf{d}, \mathbf{r})$, respectively. For any $v_{i}^{x} \in S^{1}$, we distinguish two distinct paths in $\Gamma$ terminating at $v_{i}^{x}$, denoted $l^{+}\left(v_{i}^{x}\right)$ and $l^{-}\left(v_{i}^{x}\right)$. The path $l^{\delta}\left(v_{i}^{x}\right)$ is determined by the following two conditions:

- the tail $t l^{\delta}\left(v_{i}^{x}\right)$ is in $S^{0}$ and

- $\epsilon\left(v_{i}^{x}, c\left(f_{j}^{a}\right)\right)=\delta$, where $f_{j}^{a}$ is the arrow in $l^{\delta}\left(v_{i}^{x}\right)$ incident to $v_{i}^{x}$.

Notice that this indeed defines two distinct paths, since in $\Gamma$ each vertex is incident to precisely two colored arrows whose signs under $\epsilon$ are different. We now consider the modules

$$
\begin{aligned}
& P_{0}=\bigoplus_{v_{i}^{x} \in S^{0}} P\left(v_{i}^{x}\right), \\
& P_{1}=\bigoplus_{v_{i}^{x} \in S^{1}} P\left(v_{i}^{x}\right)
\end{aligned}
$$

with a map $F: P_{1} \rightarrow P_{0}$ defined in the following way. Let $\left.F\right|_{(i, x)}: P\left(v_{i}^{x}\right) \rightarrow$ $P\left(t l^{+}\left(v_{i}^{x}\right)\right) \oplus P\left(t l^{-}\left(v_{i}^{x}\right)\right)\left(\right.$ or $\left.F\right|_{(i, x)}: P\left(v_{i}^{x}\right) \rightarrow P\left(t l^{+}\left(v_{i}^{x}\right)\right)$ when $\left.t l^{+}\left(v_{i}^{x}\right)=t l^{-}\left(v_{i}^{x}\right)\right)$ be the map given by

a. If there is a band $b \in B$ with $\Theta(b)=v_{i}^{x}$, and $t l^{+}\left(v_{i}^{x}\right)=t l^{-}\left(v_{i}^{x}\right)$,

$$
P\left(v_{i}^{x}\right) \stackrel{\left[\lambda_{b} \cdot \pi\left(l^{+}\left(v_{i}^{x}\right)\right)-\pi\left(l^{-}\left(v_{i}^{x}\right)\right)\right]}{\longrightarrow} P\left(t l^{+}\left(v_{i}^{x}\right)\right) .
$$

b. If there is a band $b \in B$ with $\Theta(b)=v_{i}^{x}$, and $t l^{+}\left(v_{i}^{x}\right) \neq t l^{-}\left(v_{i}^{x}\right)$,

$$
P\left(v_{i}^{x}\right) \stackrel{\left[\begin{array}{c}
\lambda_{b} \cdot \pi\left(l^{+}\left(v_{i}^{x}\right)\right) \\
-\pi\left(l^{-}\left(v_{i}^{x}\right)\right)
\end{array}\right]}{\longrightarrow} P\left(t l^{+}\left(v_{i}^{x}\right)\right) \oplus P\left(t l^{-}\left(v_{i}^{x}\right)\right) .
$$

c. Otherwise,

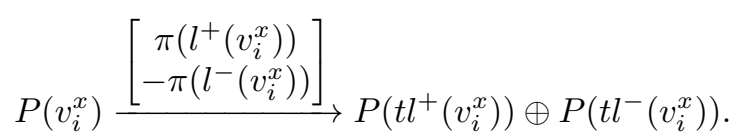

Following $\left.F\right|_{(i, x)}$ by the inclusion gives a map $P\left(v_{i}^{x}\right) \rightarrow P_{0}$ which we denote with the same symbol. Then $F$ is defined to be the map $\left.\sum_{v_{i}^{x} \in S^{1}} F\right|_{(i, x)}$.

In [1] it was shown that $P_{1} \rightarrow P_{0} \rightarrow X_{\underline{\lambda}} \rightarrow 0$ is a minimal projective resolution of $X_{\underline{\lambda}}$. In particular, $X_{\underline{\lambda}}$ has projective dimension one for all $\underline{\lambda} \in\left(k^{*}\right)^{B}$. One important consequence is the following.

Proposition 11. If $M(\mathbf{d}, \mathbf{r}, \lambda)$ is a generic indecomposable (regular) module, then it is Schur.

Proof. Suppose $M=M(\mathbf{d}, \mathbf{r}, \lambda)$ is indecomposable regular. As remarked, its projective dimension is 1 , so $\langle\langle\mathbf{d}, \mathbf{d}\rangle\rangle=\operatorname{dim}_{k} \operatorname{Hom}_{A}(M, M)-\operatorname{dim}_{k} \operatorname{Ext}_{A}^{1}(M, M)$. 
Putting this together with Corollary [10 and equation (3) yields:

$$
0=\langle\langle\mathbf{d}, \mathbf{d}\rangle\rangle=\operatorname{dim}_{k} \operatorname{Hom}_{A}(M, M)-1 .
$$

The proposition follows immediately.

Corollary 12. Let $\bmod (A, \mathbf{d}, \mathbf{r})$ be an indecomposable irreducible regular component of $\bmod (A, \mathbf{d})$. Then the following hold:

1. The field of rational invariants $k(\bmod (A, \mathbf{d}, \mathbf{r}))^{\mathrm{GL}(\mathbf{d})}$ is purely transcendental of degree 1.

2. If $\theta$ is a weight such that $\bmod (A, \mathbf{d}, \mathbf{r})_{\theta}^{s} \neq \emptyset$, then $\mathcal{M}(\bmod (A, \mathbf{d}, \mathbf{r}))_{\theta}^{s s} \cong \mathbb{P}^{1}$.

Proof. Part 1 follows immediately from the proposition above and Proposition 7(3). As for part 2, we have already mentioned that $\bmod (A, \mathbf{d}, \mathbf{r})$ is normal, so $\mathcal{M}(\bmod (A, \mathbf{d}, \mathbf{r}))_{\theta}^{s s}$ is either a point or $\mathbb{P}^{1}$ by Proposition $7(2)$. But from part (1) and Remark 3, the moduli space is not a point.

It turns out that we can actually extend the second part of the previous result to the case of triangular string algebras:

Corollary 13. Let $A=k Q / I$ be a triangular string algebra, $\mathbf{d}$ a generic root of $A$, and $C \subseteq \bmod (A, \mathbf{d})$ an indecomposable irreducible component. If $\theta \in \mathbb{Z}^{Q_{0}}$ is an integral weight such that $C_{\theta}^{s} \neq \emptyset$, then $\mathcal{M}(C)_{\theta}^{s s}$ is either a point or $\mathbb{P}^{1}$.

Proof. It has been proved in [13, Proposition 2.8] that there exists a coloring $c$ of $Q$ such that $I_{c} \subseteq I$ and $A^{\prime}=k Q / I_{c}$ is a colored gentle algebra. Hence, there exists an irreducible component $C^{\prime} \subseteq \bmod \left(A^{\prime}, \mathbf{d}\right)$ that contains $C$. Note that since any $\theta$-stable module is a Schur module and $\emptyset \neq C_{\theta}^{s} \subseteq\left(C^{\prime}\right)_{\theta}^{s}$, it follows that $C^{\prime}$ is a Schur component.

If $C$ is an orbit closure, then the moduli space in question is just a point. The other case left is when $\mathcal{M}(C)_{\theta}^{s s}$ is a rational projective curve (see Proposition $7(2)$ ). In this case, $C$ is a regular Schur component and so must be $C^{\prime}$. Indeed, if $C^{\prime}$ were not a regular component, then $C^{\prime}$ would be the closure of the orbit of a Schur module $M^{\prime}$ containing another Schur module $M^{\prime \prime}$ not isomorphic to $M^{\prime}$. But this would imply that $1=\operatorname{dim}_{k} \operatorname{End}_{A}\left(M^{\prime \prime}\right)>\operatorname{dim}_{k} \operatorname{End}_{A}\left(M^{\prime}\right)=1$, which is a contradiction. So, $C^{\prime}$ is indeed a regular Schur component with $\left(C^{\prime}\right)_{\theta}^{s} \neq \emptyset$, and hence $\mathcal{M}\left(C^{\prime}\right)_{\theta}^{s s} \simeq \mathbb{P}^{1}$ by Corollary 12 ,

Now, let $\pi: C_{\theta}^{s s} \rightarrow \mathcal{M}(C)_{\theta}^{s s}$ and $\pi^{\prime}: C_{\theta}^{\prime s s} \rightarrow \mathcal{M}\left(C^{\prime}\right)_{\theta}^{s s}$ be the quotient morphisms for the actions of PGL(d) on $C_{\theta}^{s s}$ and $C_{\theta}^{\prime s s}$, respectively. Now, consider the PGL(d)invariant morphism $\varphi: C_{\theta}^{s s} \rightarrow \mathcal{M}\left(C^{\prime}\right)_{\theta}^{s s}$ that sends $M$ to $\pi^{\prime}(M)$. From the universal property of GIT quotients, we know that there exists a morphism $f: \mathcal{M}(C)_{\theta}^{s s} \rightarrow$ $\mathcal{M}\left(C^{\prime}\right)_{\theta}^{s s}$ such that $f \circ \pi=\varphi$.

It is easy to see that $f$ is injective: Indeed, let $M_{1}, M_{2} \in C_{\theta}^{s s}$ be so that $f\left(\pi\left(M_{1}\right)\right)=f\left(\pi\left(M_{2}\right)\right)$. Then, $\pi^{\prime}\left(M_{1}\right)=\pi^{\prime}\left(M_{2}\right)$, which is equivalent to $\overline{\mathrm{GL}(\mathbf{d}) M_{1}} \cap$ $\overline{\mathrm{GL}(\mathbf{d}) M_{2}} \cap C_{\theta}^{\prime s s} \neq \emptyset$. Observe that $C_{\theta}^{s s}$ is closed in $C_{\theta}^{\prime s s}$, and hence $\overline{\mathrm{GL}(\mathbf{d}) M_{i}} \cap C_{\theta}^{\prime s s}$, $i=1,2$, are contained in $C_{\theta}^{s s}$. So, $\overline{\mathrm{GL}(\mathbf{d}) M_{1}} \cap \overline{\mathrm{GL}(\mathbf{d}) M_{2}} \cap C_{\theta}^{s s} \neq \emptyset$, which is equivalent to $\pi\left(M_{1}\right)=\pi\left(M_{2}\right)$.

Moreover, it is clear now that $f$ is surjective since otherwise $\operatorname{Im} f$ would be just a point. In conclusion, $f$ is a bijective morphism whose target variety is normal, and hence it has to be an isomorphism by (a consequence of) the Zariski's Main Theorem (in characteristic zero). 


\section{Proofs of the main Results}

We first prove Theorem 1, and then proceed with the proof of our constructive Theorem 2 .

Proof of Theorem 1. (1) Using Corollary 10, we know that we can write the generic decomposition of $C$ as

$$
C=\overline{\bigoplus_{i=1}^{n} C_{i}^{\oplus m_{i}} \oplus \bigoplus_{j=n+1}^{l} C_{j},}
$$

where $C_{i} \subseteq \bmod \left(A, \mathbf{d}_{i}\right), 1 \leq i \leq l$, are indecomposable irreducible regular components, $C_{n+1}, \ldots, C_{l}$ are orbit closures, $m_{1}, \ldots, m_{n}$ are positive integers, and $\mathbf{d}_{i} \neq \mathbf{d}_{j}, \forall 1 \leq i \neq j \leq n$. (Of course, we do allow $n=0$ or $l=n$.)

But note that for each $1 \leq i \leq n, k\left(C_{i}\right)^{\mathrm{GL}\left(\mathbf{d}_{i}\right)} \simeq k(t)$ by Corollary 12(1). Now applying Proposition 4, we obtain that $k(C)^{\mathrm{GL}(\mathbf{d})} \simeq k\left(t_{1}, \ldots, t_{N}\right)$, where $N=$ $\sum_{i=1}^{n} m_{i}$.

(2) First of all, any $\theta$-semi-stable irreducible component $C \subseteq \bmod (A, \mathbf{d})$ is $\theta$ well-behaved. This follows immediately from the uniqueness property in Corollary 10 .

Now, let us assume that $C$ is a $\theta$-semi-stable irreducible regular component whose $\theta$-stable decomposition is of the form

$$
C=C_{1} \dot{+} \ldots \dot{+} C_{l}
$$

with $C_{1}, \ldots, C_{l}$ indecomposable irreducible regular components. Using Corollary 10 again, we can write the $\theta$-stable decomposition of $C$ as

$$
C=l_{1} \cdot C_{1} \dot{+} \ldots \dot{+} l_{m} \cdot C_{m},
$$

where $C_{i} \subseteq \bmod \left(A, \mathbf{d}_{i}\right), 1 \leq i \leq m$, are $\theta$-stable irreducible regular components, and $\mathbf{d}_{i} \neq \mathbf{d}_{j}$ for all $1 \leq i \neq j \leq m$.

Moreover, if $C^{\prime} \subseteq \bmod (A, \mathbf{d})$ is an irreducible component containing $\overline{\bigoplus_{i=1}^{m} C_{i}^{\oplus l_{i}}}$ then $C^{\prime}$ contains a regular module, call it $M_{0}$. We next claim that $C^{\prime}$ is a regular component. Indeed, consider the non-empty open subset $U$ of $C^{\prime}$ consisting of those modules $M \in C^{\prime}$ for which $\operatorname{rank} M(a)$ is maximal for all arrows $a \in Q_{1}$. Then, for any module $M \in U$, we have

$$
\operatorname{dim}_{k} M_{0}=\sum_{a \in Q_{1}} \operatorname{rank} M_{0}(a) \leq \sum_{a \in Q_{1}} \operatorname{rank} M(a)=\operatorname{dim}_{k} M-s,
$$

where $s$ is the number of string indecomposable modules occurring in a direct sum decomposition of $M$ into indecomposables. (This rightmost identity above is known to hold for any module over a string algebra; it follows from the construction of the band and string modules.) Consequently, $s=0$ and hence the generic module in $C^{\prime}$ is regular. It now follows from Corollary 10 that $C=C^{\prime}$. At this point, we can apply Theorem 6 and Corollary $12(2)$ and deduce that $\mathcal{M}(C)_{\theta}^{s s} \simeq \prod_{i=1}^{m} \mathbb{P}^{l_{i}}$.

To prove Theorem 2, we will use the projective resolution from Section 5.2, But first let us briefly recall the construction of the so-called generalized Schofield semiinvariants on module varieties following Derksen and Weyman [21], and Domokos [22].

Let $X$ be an $A$-module and $P_{1} \stackrel{F}{\rightarrow} P_{0} \rightarrow X \rightarrow 0$ be a fixed minimal projective presentation of $X$ in $\bmod (A)$. Let $\theta^{X} \in \mathbb{Z}^{Q_{0}}$ be the integral weight defined so 
that $\theta^{X}(x)$ is the multiplicity of $P_{x}$ in $P_{0}$ minus the multiplicity of $P_{x}$ in $P_{1}$ for all $x \in Q_{0}$. For an arbitrary $A$-module $M$, we have

$$
\begin{aligned}
\theta^{X}(\operatorname{dim} M) & =\operatorname{dim}_{k} \operatorname{Hom}_{A}\left(P_{0}, M\right)-\operatorname{dim}_{k} \operatorname{Hom}_{A}\left(P_{1}, M\right) \\
& =\operatorname{dim}_{k} \operatorname{Hom}_{A}(X, M)-\operatorname{dim}_{k} \operatorname{Hom}_{A}(M, \tau X) .
\end{aligned}
$$

Here, $\tau X$ is the Auslander-Reiten translation of $X$ given by $D\left(\right.$ Coker $\left.f^{t}\right)$, where $(-)^{t}=\operatorname{Hom}_{A}(-, A)$ and $D=\operatorname{Hom}_{k}(-, k)$ (for more details, see [3, §IV.2]). Notice that if $\operatorname{pdim} X \leq 1$, then

$$
\theta^{X}(\operatorname{dim} M)=\operatorname{dim}_{k} \operatorname{Hom}_{A}(X, M)-\operatorname{dim}_{k} \operatorname{Ext}_{A}^{1}(X, M)=\langle\langle\operatorname{dim} X, \operatorname{dim} M\rangle\rangle .
$$

Before we continue our discussion on Schofield semi-invariants we mention the following example of a weight that satisfies the conditions in Theorem 1(2):

Example 2. Let $C \subseteq \bmod (A, \mathbf{d})$ be an irreducible regular component. Then, its generic decomposition is of the form

$$
C=\overline{\bigoplus_{i=1}^{m} C_{i}^{l_{i}}},
$$

where $C_{i} \subseteq \bmod \left(A, \mathbf{d}_{i}\right), 1 \leq i \leq m$, are indecomposable irreducible regular components and $\mathbf{d}_{i} \neq \mathbf{d}_{j}, \forall 1 \leq i \neq j \leq m$. Notice also that $\left\langle\left\langle\mathbf{d}_{i}, \mathbf{d}_{i}\right\rangle\right\rangle=0$ for all $1 \leq i, j \leq m$ (for details, see Lemma 15).

We claim that the weight $\theta:=\langle\langle\mathbf{d}, \cdot\rangle\rangle$ satisfies the conditions in Theorem 11(2). Specifically, we claim that the $\theta$-stable decomposition of $C$ is precisely

$$
C=l_{1} \cdot C_{1} \dot{+} \ldots \dot{+} l_{m} \cdot C_{m} .
$$

For this, it is clearly enough to show that $C_{i, \theta}^{s} \neq \emptyset$ for each $1 \leq i \leq m$. Let $M_{i} \in C_{i}$ be a Schur homogeneous module (e.g. choose $M_{i}$ to be any indecomposable band module in $C_{i}$ ). Let us check that $M_{i} \in C_{i, \theta}^{s}$. Set $M:=\bigoplus_{i=1}^{m} M_{i}^{l_{i}}$ and note that $\operatorname{pdim} M \leq 1$ and $\tau M \simeq M$ since each $M_{i}$ has these two properties. From the discussion above, we have that $\theta=\theta^{M}$, and so

$$
\theta\left(\operatorname{dim} M_{i}^{\prime}\right)=\operatorname{dim}_{K} \operatorname{Hom}_{A}\left(M, M_{i}^{\prime}\right)-\operatorname{dim}_{K} \operatorname{Hom}_{A}\left(M_{i}^{\prime}, M\right),
$$

for any proper submodule $M_{i}^{\prime}<M_{i}$. Using the fact that $M_{i}$ is $\operatorname{Schur}, \operatorname{Hom}_{A}\left(M_{j}, M_{l}\right)$ $=0$ for all $j \neq l$, and $M_{i}^{\prime} \neq M_{i}$, it is easy to see that $\operatorname{Hom}_{A}\left(M, M_{i}^{\prime}\right)=0$ and hence

$$
\theta\left(\operatorname{dim} M_{i}^{\prime}\right)=-\operatorname{dim}_{K} \operatorname{Hom}_{A}\left(M_{i}^{\prime}, M\right)<0 .
$$

This shows that $C$ has the desired $\theta$-stable decomposition. We can now apply Theorem 1(2) and conclude that $\mathcal{M}(C)_{\theta}^{s s} \simeq \prod_{i=1}^{m} \mathbb{P}^{l_{i}}$.

Let $\mathbf{d} \in \mathbb{Z}^{Q_{0}}$ be a dimension vector of $A$ such that $\theta^{X}(\mathbf{d})=0$. Then for any module $M \in \bmod (A, \mathbf{d}), \operatorname{dim}_{k} \operatorname{Hom}_{A}\left(P_{0}, M\right)=\operatorname{dim}_{k} \operatorname{Hom}_{A}\left(P_{1}, M\right)$, and hence the linear map

$$
\operatorname{Hom}_{A}(F, M): \operatorname{Hom}_{A}\left(P_{0}, M\right) \rightarrow \operatorname{Hom}_{A}\left(P_{1}, M\right)
$$

can be viewed as a square matrix. We can therefore define

$$
\bar{c}^{X}: \bmod (A, \mathbf{d}) \rightarrow k, \quad \bar{c}^{X}(M)=\operatorname{det} \operatorname{Hom}_{A}(F, M) .
$$

It is easy to see that $\bar{c}^{X}$ is a semi-invariant of weight $\theta^{X}$. Moreover, any other choice of a minimal projective presentation of $X$ leads to the same semi-invariant, up to a non-zero scalar. We call $\bar{c}^{X}$ a generalized Schofield semi-invariant. 
We can now apply this setup to triangular gentle algebras $A=k Q / I_{c}$, when $X=M(\mathbf{d}, \mathbf{r}, \lambda)$, a generic indecomposable regular module. Let

$$
\bmod (A, \mathbf{d}, \mathbf{r})=\overline{\bigcup_{\lambda \in k^{*}} \mathrm{GL}(\mathbf{d}) \cdot M(\mathbf{d}, \mathbf{r}, \lambda)}
$$

be an indecomposable irreducible regular component. Evidently, $M(\mathbf{d}, \mathbf{r}, \lambda)$ has projective dimension 1, and from Corollary 10 we have that $\langle\langle\mathbf{d}, \mathbf{d}\rangle\rangle=0$. Therefore, $\theta^{M(\mathbf{d}, \mathbf{r}, \lambda)}(\mathbf{d})=0$ and $\bar{c}^{M(\mathbf{d}, \mathbf{r}, \lambda)}(M)=\operatorname{det} \operatorname{Hom}_{A}(F, M)$ for all $M \in \bmod (A, \mathbf{d}, \mathbf{r})$, where $P_{1} \stackrel{F}{\rightarrow} P_{0} \rightarrow M(\mathbf{d}, \mathbf{r}, \lambda)$ is the minimal projective resolution described in Section 5.2 .

Proposition 14. Let $\bmod (A, \mathbf{d}, \mathbf{r})=\overline{\bigcup_{\lambda \in k^{*}} \mathrm{GL}(d) \cdot M(\mathbf{d}, \mathbf{r}, \lambda)}$ be an indecomposable irreducible regular component of $\bmod (A, \mathbf{d})$. Then up to sign

$$
\bar{c}^{M(\mathbf{d}, \mathbf{r}, \lambda)}(M(\mathbf{d}, \mathbf{r}, \mu))=\lambda^{p} \mu^{l}(\lambda-\mu)^{q}
$$

for some integers $p=p(\mathbf{d}, \mathbf{r}), l=l(\mathbf{d}, \mathbf{r}), q=q(\mathbf{d}, \mathbf{r})$.

Proof. Since the projective dimension of $M(\mathbf{d}, \mathbf{r}, \lambda)$ is one, $\bar{c}^{M(\mathbf{d}, \mathbf{r}, \lambda)}(M(\mathbf{d}, \mathbf{r}, \mu))$ is non-zero precisely when $\operatorname{Ext}_{A}^{1}(M(\mathbf{d}, \mathbf{r}, \lambda), M(\mathbf{d}, \mathbf{r}, \mu)) \neq 0$. Consider the regular map $k^{*} \times k^{*} \rightarrow k$ defined by sending $(\lambda, \mu)$ to $\bar{c}^{M(\mathbf{d}, \mathbf{r}, \lambda)}(M(\mathbf{d}, \mathbf{r}, \mu))$. By equation (2), this map takes value zero precisely when $\lambda=\mu$. Therefore, the only factors of $\bar{c}^{M(\mathbf{d}, \mathbf{r}, \lambda)}(M(\mathbf{d}, \mathbf{r}, \mu))$ that are possible are $\lambda, \mu$ and $\lambda-\mu$. The formula follows immediately.

We now extend this formula to the general case, i.e., when $\bmod (A, \mathbf{d}, \mathbf{r})$ is an irreducible regular component (not necessarily indecomposable).

Lemma 15. Suppose $\bmod (A, \mathbf{d}, \mathbf{r})=\overline{\bigoplus_{i=1}^{n} \bmod \left(A, \mathbf{d}_{i}, \mathbf{r}_{i}\right)^{m_{i}}}$ is the generic decomposition of the irreducible regular component $\bmod (A, \mathbf{d}, \mathbf{r})$. Then for each $i=$ $1, \ldots, n,\left\langle\left\langle\mathbf{d}_{i}, \mathbf{d}\right\rangle\right\rangle=0$.

Proof. Recall from equation (2) that $\operatorname{Ext}_{A}^{1}\left(M(\mathbf{d}, \mathbf{r}, \underline{\lambda}), M\left(\mathbf{d}, \mathbf{r}, \underline{\lambda^{\prime}}\right)\right)=0$ when $\underline{\lambda}, \underline{\lambda^{\prime}}$ share no common components. In particular, $\operatorname{Ext}_{A}^{1}\left(M\left(\mathbf{d}_{i}, \mathbf{r}_{i}, \lambda_{i}\right), M\left(\mathbf{d}_{j}, \mathbf{r}_{j}, \lambda_{j}\right)\right)=0$ for $i \neq j, \lambda_{i} \neq \lambda_{j} \in k^{*}$ (if $\lambda_{i}=\lambda_{j}$ and $\Gamma\left(\mathbf{d}_{i}, \mathbf{r}_{i}\right)$ is of the same shape as $\Gamma\left(\mathbf{d}_{j}, \mathbf{r}_{j}\right)$, then there are indeed extensions). Moreover, by definition $\bmod \left(A, \mathbf{d}_{i}, \mathbf{r}_{i}\right)$ is a regular irreducible component, so $\left\langle\left\langle\mathbf{d}_{i}, \mathbf{d}_{i}\right\rangle\right\rangle=0$.

Let $\left\{\lambda_{j} ; j=1, \ldots, n\right\}$ be a collection of pairwise distinct elements in $k^{*}$, and abbreviate $M\left(\mathbf{d}_{j}, \mathbf{r}_{j}, \lambda_{j}\right)$ by $X^{(j)}$. Then for any given $i,\left\langle\left\langle\mathbf{d}_{i}, \mathbf{d}\right\rangle\right\rangle \geq 0$ since

$$
\begin{aligned}
\left\langle\left\langle\mathbf{d}_{i}, \mathbf{d}\right\rangle\right\rangle & =\sum_{j=1}^{n} m_{j}\left\langle\left\langle\mathbf{d}_{i}, \mathbf{d}_{j}\right\rangle\right\rangle \\
& =\sum_{j \neq i} m_{j}\left\langle\left\langle\mathbf{d}_{i}, \mathbf{d}_{j}\right\rangle\right\rangle \\
& =\sum_{j \neq i} m_{j}\left(\operatorname{dim} \operatorname{Hom}\left(X^{(i)}, X^{(j)}\right)-\operatorname{dim} \operatorname{Ext}_{A}^{1}\left(X^{(i)}, X^{(j)}\right)\right) \\
& =\sum_{j \neq i} m_{j} \operatorname{dim} \operatorname{Hom}_{A}\left(X^{(i)}, X^{(j)}\right) \geq 0 .
\end{aligned}
$$

However, from Corollary 10, $0=\langle\langle\mathbf{d}, \mathbf{d}\rangle\rangle=\sum_{i=1}^{n} m_{i}\left\langle\left\langle\mathbf{d}_{i}, \mathbf{d}\right\rangle\right\rangle$, and so $\left\langle\left\langle\mathbf{d}_{i}, \mathbf{d}\right\rangle\right\rangle=0$ since $m_{i} \geq 1$. 
Remark 6. Given this proposition, the generalized Schofield semi-invariant $\bar{c}^{M\left(\mathbf{d}_{i}, \mathbf{r}_{i}, \lambda\right)}$ is a non-zero semi-invariant function on the regular component $\bmod (A, \mathbf{d}, \mathbf{r})$.

Proposition 16. Suppose again that $\bmod (A, \mathbf{d}, \mathbf{r})=\overline{\bigoplus_{i=1}^{n} \bmod \left(A, \mathbf{d}_{i}, \mathbf{r}_{i}\right)^{\oplus m_{i}}}$ is the generic decomposition of the irreducible regular component $\bmod (A, \mathbf{d}, \mathbf{r})$, and for $\mu=\left(\mu(i, j) ; i=1, \ldots, n, j=1, \ldots, m_{i}, \mu(i, j) \in k^{*}\right)$ consider the generic module

$$
X_{\underline{\mu}}=\bigoplus_{i=1}^{n}\left(\bigoplus_{j=1}^{m_{i}} M\left(\mathbf{d}_{i}, \mathbf{r}_{i}, \mu(i, j)\right)\right)
$$

Then for any $\lambda \in k^{*}$,

$$
\bar{c}^{M\left(\mathbf{d}_{i}, \mathbf{r}_{i}, \lambda\right)}\left(X_{\underline{\mu}}\right)=\left[\prod_{j=1}^{m_{i}} \lambda^{p_{i}} \mu(i, j)^{l_{i}}(\lambda-\mu(i, j))^{q_{i, j}}\right] \cdot\left[\prod_{i^{\prime} \neq i} \prod_{j=1}^{m_{i^{\prime}}} \lambda^{p_{i^{\prime}}} \mu\left(i^{\prime}, j\right)^{l_{i^{\prime}}}\right]
$$

for some integers $p_{i}, l_{i}, q_{i, j}$.

Remark 7. In [12, Chapter 4.2], the first author uses explicit matrix calculations to show that the exponents $q_{i, j}$ in the above proposition are equal to one. Although it is not a necessity for the forthcoming proofs, we will use the exponent one for readability.

Proof. First, since $M\left(\mathbf{d}_{i}, \mathbf{r}_{i}, \lambda\right)$ has projective dimension one, and $\left\langle\left\langle\mathbf{d}_{i}, \mathbf{d}\right\rangle\right\rangle=0$, $c^{M\left(\mathbf{d}_{i}, \mathbf{r}_{i}, \lambda\right)}\left(X_{\mu}\right)$ is a well-defined semi-invariant which is non-zero precisely when $\operatorname{Ext}_{A}^{1}\left(M\left(\mathbf{d}_{i}, \overline{\mathbf{r}}_{i}, \lambda\right), X_{\mu}\right)=0$. Consider again the regular map $k^{*} \times\left(k^{*}\right)^{\sum m_{i}} \rightarrow k$ defined by sending $(\bar{\lambda}, \mu)$ to $\bar{c}^{M\left(\mathbf{d}_{i}, \mathbf{r}_{i}, \lambda\right)}\left(X_{\mu}\right)$. From equations (2) and (3), this map takes value zero precisely when $\lambda=\mu(i, \bar{j})$ for some $j=1, \ldots, m_{i}$ (i.e., when $X_{\underline{\mu}}$ has a direct summand isomorphic to $\left.M\left(\mathbf{d}_{i}, \mathbf{r}_{i}, \lambda\right)\right)$. Therefore, the only factors are $\lambda, \mu\left(i^{\prime}, j\right)$ and $\lambda-\mu(i, j)$, so indeed equation (5) holds.

This allows us to prove our main constructive theorem:

Proof of Theorem 2. We need only exhibit an open set $U \subset \bmod (A, \mathbf{d}, \mathbf{r})$ on which the functions separate orbits. In this case, 31, Lemma 2.1] implies that

$$
k(\bmod (A, \mathbf{d}, \mathbf{r}))^{\mathrm{GL}(\mathbf{d})} \cong k\left(\frac{\bar{c}^{M\left(\mathbf{d}_{i}, \mathbf{r}_{i}, \lambda(i, j)\right)}}{\bar{c}^{M\left(\mathbf{d}_{i}, \mathbf{r}_{i}, \lambda(i, j+1)\right)}} ; i=1, \ldots, n, j=0, \ldots, m_{i}-1\right) .
$$

There are clearly $N=\sum m_{i}$ such functions from which we can choose a subset that forms a transcendental basis. By Theorem 1, $N$ is the transcendence degree of $k(\bmod (A, \mathbf{d}, \mathbf{r}))^{\mathrm{GL}(\mathbf{d})}$ over $k$, but taking any fewer elements from the set will yield a smaller transcendence degree, a contradiction.

Now we prove the first claim. Notice that as the image of the dominant map $\mathrm{GL}(\mathbf{d}) \times\left(k^{*}\right)^{B} \rightarrow \bmod (A, \mathbf{d}, \mathbf{r})$, the set $\bigcup_{\mu \in\left(k^{*}\right)^{B}} \mathrm{GL}(\mathbf{d}) \cdot M(\mathbf{d}, \mathbf{r}, \mu)$ is constructible, so it contains an open dense set $U^{\prime}$ of $\bmod (A, \mathbf{d}, \mathbf{r})$. Take $U$ to be the open set $U=\bigcap_{i, j}\left\{X \in \bmod (A, \mathbf{d}, \mathbf{r}) \mid \bar{c}^{M\left(\mathbf{d}_{i}, \mathbf{r}_{i}, \lambda(i, j)\right)}(X) \neq 0\right\} \cap U^{\prime}$, and let $X_{\mu}, X_{\underline{\nu}}$ be two generic modules in $U$ such that for each $i=1, \ldots, n, j=0, \ldots, m_{i}-\overline{1}$,

$$
\frac{\bar{c}^{M\left(\mathbf{d}_{i}, \mathbf{r}_{i}, \lambda(i, j)\right)}\left(X_{\underline{\mu}}\right)}{\bar{c}^{M\left(\mathbf{d}_{i}, \mathbf{r}_{i}, \lambda(i, j+1)\right)}\left(X_{\underline{\mu}}\right)}=\frac{\bar{c}^{M\left(\mathbf{d}_{i}, \mathbf{r}_{i}, \lambda(i, j)\right)}\left(X_{\underline{\nu}}\right)}{\bar{c}^{M\left(\mathbf{d}_{i}, \mathbf{r}_{i}, \lambda(i, j+1)\right)}\left(X_{\underline{\nu}}\right)} \text {. }
$$


Expanding these by applying equation (5), for each, we have

$$
\begin{gathered}
\frac{\left[\prod_{j^{\prime}=1}^{m_{i}} \lambda(i, j)^{p_{i}} \mu\left(i, j^{\prime}\right)^{l_{i}}\left(\lambda(i, j)-\mu\left(i, j^{\prime}\right)\right)\right] \cdot\left[\prod_{i^{\prime} \neq i} \prod_{j^{\prime}=1}^{m_{i^{\prime}}} \lambda(i, j)^{p_{i^{\prime}}} \mu\left(i^{\prime}, j^{\prime}\right)^{l_{i^{\prime}}}\right]}{\left[\prod_{j^{\prime}=1}^{m_{i}} \lambda(i, j+1)^{p_{i}} \mu\left(i, j^{\prime}\right)^{l_{i}}\left(\lambda(i, j+1)-\mu\left(i, j^{\prime}\right)\right)\right] \cdot\left[\prod_{i^{\prime} \neq i}^{m_{i^{\prime}}} \lambda(i, j+1)^{p_{i^{\prime}}} \mu\left(i^{\prime}, j^{\prime}\right)^{l_{i^{\prime}}}\right]} \\
=\frac{\left[\prod_{j^{\prime}=1}^{m_{i}} \lambda(i, j)^{p_{i}} \nu\left(i, j^{\prime}\right)^{l_{i}}\left(\lambda(i, j)-\nu\left(i, j^{\prime}\right)\right)\right] \cdot\left[\prod_{i^{\prime} \neq i} \prod_{j^{\prime}=1}^{m_{i^{\prime}}} \lambda(i, j)^{p_{i^{\prime}}} \nu\left(i^{\prime}, j^{\prime}\right)^{l_{i^{\prime}}}\right]}{\left[\prod_{j^{\prime}=1}^{m_{i}} \lambda(i, j+1)^{p_{i}} \nu\left(i, j^{\prime}\right)^{l_{i}}\left(\lambda(i, j+1)-\nu\left(i, j^{\prime}\right)\right)\right] \cdot\left[\prod_{i^{\prime} \neq i}^{m_{i^{\prime}}} \lambda(i, j+1)^{p_{i^{\prime}}} \nu\left(i^{\prime}, j^{\prime}\right)^{l_{i^{\prime}}}\right]} .
\end{gathered}
$$

This is equivalent to the existence of a constant $c_{i}$ for each $i=1, \ldots, n$, with

$$
\frac{\prod_{j^{\prime}=1}^{m_{i}}\left(\lambda(i, j)-\mu\left(i, j^{\prime}\right)\right)}{\prod_{j^{\prime}=1}^{m_{i}}\left(\lambda(i, j)-\nu\left(i, j^{\prime}\right)\right)}=c_{i}
$$

for each $j=0, \ldots, m_{i}-1$. In particular, for each $i, \prod_{j^{\prime}=1}^{m_{i}}\left(x-\mu\left(i, j^{\prime}\right)\right)-$ $c_{i} \prod_{j^{\prime}=1}^{m_{i}}\left(x-\nu\left(i, j^{\prime}\right)\right)$ is a degree $m_{i}$ polynomial with $m_{i}+1$ distinct roots (by selection of the $\lambda(i, j))$, and so for all $i, c_{i}=1$. Thus, for each $i$ there is a permutation $\sigma^{(i)}$ of $\left\{1, \ldots, m_{i}\right\}$ such that $\mu\left(i, j^{\prime}\right)=\nu\left(i, \sigma^{(i)}\left(j^{\prime}\right)\right)$. In particular, $X_{\mu} \cong X_{\underline{\nu}}$.

Example 3. Finally, we point out some pathologies that show the necessity of the conditions of Proposition 4.

(i) Consider the gentle algebra $A(2)=k Q / I_{c}$ where the bound quiver and coloring are indicated below:

$$
1 \underset{-\overline{b_{1}}}{a_{1}} \overbrace{-\frac{b_{2}}{7}}^{a_{2}} 3 .
$$

Let $\mathbf{d}=(2,2,2)$ and $\mathbf{r}\left(a_{i}\right)=\mathbf{r}\left(b_{i}\right)=1$ for $i=1,2$. The generic module in this case is the module

$$
\pi_{*}\left(\begin{array}{c}
0 \longrightarrow 0-->0 \\
0-->0 \longrightarrow 0
\end{array}\right)
$$

Let $\mathbf{r}^{\prime}$ be the rank function $\mathbf{r}^{\prime}\left(a_{1}\right)=\mathbf{r}^{\prime}\left(b_{2}\right)=1$ and $\mathbf{r}^{\prime}\left(a_{2}\right)=\mathbf{r}^{\prime}\left(b_{1}\right)=0$, and $\mathbf{r}^{\prime \prime}$ be the rank function $\mathbf{r}^{\prime \prime}\left(a_{2}\right)=\mathbf{r}^{\prime \prime}\left(b_{1}\right)=1$ and $\mathbf{r}^{\prime \prime}\left(a_{1}\right)=\mathbf{r}^{\prime \prime}\left(b_{2}\right)=0$. Then the generic decomposition of $\bmod (A, \mathbf{d}, \mathbf{r})$ is

$$
\overline{\bmod \left(A,(1,1,1), \mathbf{r}^{\prime}\right) \oplus \bmod \left(A,(1,1,1), \mathbf{r}^{\prime \prime}\right)} .
$$

Thus, $\bmod (A, \mathbf{d}, \mathbf{r})$ decomposes generically into the direct sum of two distinct orbit closures of the same dimension vector. This is in contrast to the situation in which $\bmod (A, \mathbf{d}, \mathbf{r})$ is a regular component (see Corollary 10). 
(ii) Indecomposable generic modules are not necessarily Schur. Consider the representation space of the bound quiver with coloring, dimension vector, and rank sequence as depicted in the diagram

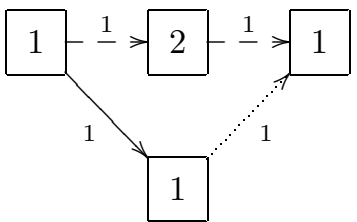

The generic module $X$ in this module variety takes the form

$$
\pi_{*}\left(\searrow_{0}^{\circ-->e_{1}-7_{0}^{0}} e_{2}\right)
$$

where $e_{1}, e_{2}$ is a basis for the 2-dimensional vector space at the second vertex in the top row. There is a non-trivial endomorphism $X \rightarrow X$ given on basis elements by $e_{2} \mapsto e_{1}$, and all other basis elements are sent to 0 .

(iii) It is possible that in $\bmod (A, \mathbf{d})$ there are two distinct indecomposable irreducible components, one of which is regular. Consider the module variety of

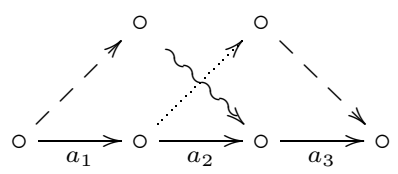

with dimension vector $\mathbf{d}=(1,1, \ldots, 1)$, and the following two rank functions: $\mathbf{r}$ defined by $\mathbf{r}\left(a_{2}\right)=0$ and all other ranks are one, and $\mathbf{r}^{\prime}$ defined by $\mathbf{r}^{\prime}\left(a_{1}\right)=\mathbf{r}^{\prime}\left(a_{3}\right)=0$ and all other ranks are one. It is clear that these are maximal with respect to $\mathbf{d}$. The generic modules are given below:

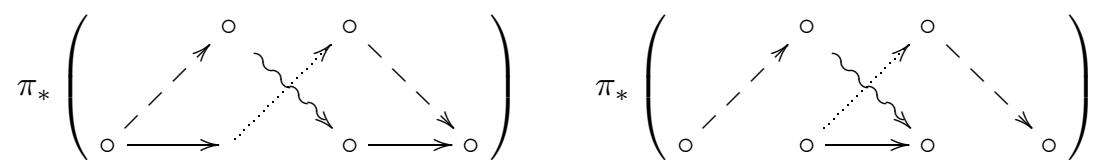

Notice that the generic module in $\bmod (\mathbf{d}, \mathbf{r})$ is an indecomposable band, and that in $\bmod \left(\mathbf{d}, \mathbf{r}^{\prime}\right)$ it is an indecomposable string.

(iv) Consider the triangular string algebra $A=k Q / I$, where $Q$ is the quiver

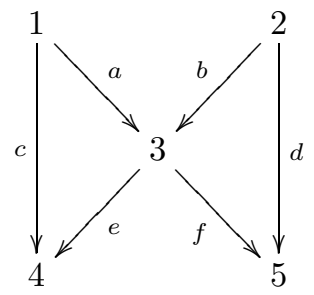

and $I$ is the ideal generated by $e a, e b, f a, f b$. We call $A$ the butterfly string algebra. Notice that $A$ is not a colored gentle algebra. 
It has been proved in [16, Section 4.1] that there is only one indecomposable irreducible regular component $C$, namely the one corresponding to the band $B=c^{-1} e f^{-1} d b^{-1} a$ and dimension vector $\mathbf{d}=(1,1,2,1,1)$, and $C$ is not a Schur component. Nonetheless, it has been proved in [16, Theorem 4.1] that for any weight $\theta \in \mathbb{Z}^{5}$ with $C_{\theta}^{s s} \neq \emptyset$, the moduli space $\mathcal{M}(C)_{\theta}^{s s}$ is just a point.

\section{Concluding Remarks}

We conclude this section by putting these results in the the broader context of a program aimed at characterizing tame algebras and parametrizing modules over such algebras using invariant-theoretic techniques. It was Jerzy Weyman in private communication who first made the following conjecture.

Conjecture 17 (Weyman). Let $(Q, I)$ be a bound quiver, and $A=k Q / I$ its bound quiver algebra. Then the following are equivalent:

(1) A is of tame representation type;

(2) for any irreducible component $C \subset \bmod (A, \mathbf{d})$ and any weight $\theta$ such that $C_{\theta}^{s s} \neq \emptyset, \mathcal{M}(C)_{\theta}^{s s}$ is a product of projective spaces.

The implication $(2) \Longrightarrow(1)$ of this conjecture has been verified for the class of quasi-tilted algebras and of strongly simply connected algebras (see [15]).

The other implication $(1) \Longrightarrow(2)$ of the conjecture above has been verified in 15] for quasi-tilted algebras when the irreducible components involved are isotropic. Since gentle algebras are tame, Theorem 1(2) proves this implication over regular irreducible components for triangular gentle algebras. In order to prove it over arbitrary irreducible components, one would need to have a better understanding of the $\theta$-stable decomposition of irreducible components of module varieties. We plan to address this in future work.

A particular case of Conjecture 17 is:

Conjecture 18. Let $A$ be a tame algebra, $\mathbf{d}$ a generic root of $A$, and $C \subseteq$ $\bmod (A, \mathbf{d})$ an indecomposable irreducible component. Then, for any weight $\theta$ for which $C_{\theta}^{s s} \neq \emptyset$, the moduli space $\mathcal{M}(C)_{\theta}^{s s}$ is either a point or $\mathbb{P}^{1}$.

Notice that Proposition 7 and Corollary 13 offer evidence for this conjecture. In fact, a proof of it for triangular string algebras would follow from Corollary 13 and a positive resolution to the following question:

Question 19. Let $A$ be a tame algebra, $\mathbf{d}$ a generic root, and $C \subseteq \bmod (A, \mathbf{d})$ an indecomposable irreducible component. If $\operatorname{dim} M(C)_{\theta}^{s s}=1$, is it true that $C_{\theta}^{s} \neq \emptyset$ ?

This question has a positive answer when $A$ is a tame quasi-tilted algebra, or a triangular gentle algebra, or the butterfly string algebra from Example 3 .

The birational analogue of Conjecture 17 would be the following, suggested by the second author.

Conjecture 20. Let $(Q, I)$ be a bound quiver, and $A=k Q / I$ its bound quiver algebra.

(a) The following are equivalent:

(1) A is of tame representation type; 
(2) for every irreducible indecomposable component $C \subset \bmod (A, \mathbf{d})$, $k(C)^{\mathrm{GL}(\mathbf{d})}$ is a rational field of transcendence degree at most one, that $i s, k$ or $k(t)$;

(b) assume that $A$ is a tame algebra. Then, for any irreducible component $C \subseteq \bmod (A, \mathbf{d}), k(C)^{\mathrm{GL}(\mathbf{d})}$ is a rational field over $k$.

Note that condition (2) of the conjecture above simply says that for an indecomposable irreducible component $C \subseteq \bmod (A, \mathbf{d})$ the rational quotient $C / \mathrm{GL}(\mathbf{d})$ is either a point or just $\mathbb{P}^{1}$, and this is very much in sync with the philosophy behind the tameness of an algebra.

Conjecture 20 has been verified for the class of quasi-tilted algebras (see [14, 15]). Moreover, our Theorem 1 is a verification of Conjecture 20 for the class of triangular gentle algebras.

The implication $(2) \Longrightarrow(1)$ has also been verified for the class of strongly simply connected algebras (see [15]). We plan to use the methods of this paper to settle this conjecture for the class of strongly simply connected algebras.

A separate and still difficult problem in general is the determination of a transcendental basis of $k(C)^{\mathrm{GL}(\mathbf{d})}$. This was achieved by Ringel in the tame hereditary case [36, but in general such a basis is unknown. Our Theorem 2 gives such a basis in the case when $A$ is a triangular gentle algebra and $C$ is an irreducible regular component.

\section{ACKNowledGements}

The authors would like to thank Harm Derksen and Piotr Dowbor for inspiring discussions on the subject of the paper. The authors are also very thankful to the referee for a very thorough report on the paper and for providing the elegant arguments in the proof of Propositions 14 and 16.

\section{REFERENCES}

[1] Daniele Arcara, Aaron Bertram, Izzet Coskun, and Jack Huizenga, The minimal model program for the Hilbert scheme of points on $\mathbb{P}^{2}$ and Bridgeland stability, Adv. Math. 235 (2013), 580-626, DOI 10.1016/j.aim.2012.11.018. MR.3010070

[2] Ibrahim Assem, Thomas Brüstle, Gabrielle Charbonneau-Jodoin, and Pierre-Guy Plamondon, Gentle algebras arising from surface triangulations, Algebra Number Theory 4 (2010), no. 2, 201-229, DOI 10.2140/ant.2010.4.201. MR2592019(2011h:16045)

[3] Ibrahim Assem, Daniel Simson, and Andrzej Skowronski, Elements of the representation theory of associative algebras. Vol. 1, London Mathematical Society Student Texts, vol. 65, Cambridge University Press, Cambridge, 2006. Techniques of representation theory. MR 2197389 (2006j:16020)

[4] Grzegorz Bobiński, On the zero set of semi-invariants for regular modules over tame canonical algebras, J. Pure Appl. Algebra 212 (2008), no. 6, 1457-1471, DOI 10.1016/j.jpaa.2007.10.002. MR2391660(2009c:16042)

[5] Grzegorz Bobiński and Andrzej Skowroński, Geometry of directing modules over tame algebras, J. Algebra 215 (1999), no. 2, 603-643, DOI 10.1006/jabr.1998.7748. MR.1686208 (2000f:16017)

[6] Grzegorz Bobiński and Andrzej Skowroński, Geometry of modules over tame quasi-tilted algebras, Colloq. Math. 79 (1999), no. 1, 85-118. MR.1671811(2000i:14067)

[7] Grzegorz Bobiński and Andrzej Skowroński, Geometry of periodic modules over tame concealed and tubular algebras, Algebr. Represent. Theory 5 (2002), no. 2, 187-200, DOI 10.1023/A:1015606729502. MR:1909550(2003d:16021)

[8] Ch. Böhning, The rationality problem in invariant theory. Preprint, arXiv:0904.0899v1 [math.AG], 2009. 
[9] M. Brion, Invariants et covariants des groupes algébriques réductifs. In Théorie des invariants et Géométrie des variétés quotients, volume 61 of Travaux en cours. Herman, 2010.

[10] M. C. R. Butler and Claus Michael Ringel, Auslander-Reiten sequences with few middle terms and applications to string algebras, Comm. Algebra 15 (1987), no. 1-2, 145-179, DOI 10.1080/00927878708823416. MR876976 (88a:16055)

[11] A. T. Carroll, Generic modules for string algebras. Preprint available at arXiv:1111.5064 [math.RT], 2011.

[12] A. T. Carroll, Semi-Invariants for Gentle String Algebras, ProQuest LLC, Ann Arbor, MI, 2012. Thesis (Ph.D.)-Northeastern University. MR3004358

[13] A. T. Carroll and J. Weyman, Semi-invariants for gentle algebras. Contemporary Mathematics (to appear). Preprint available at arXiv:1106.0774 [math.RT], 2013.

[14] C. Chindris, Geometric characterizations of the representation type of hereditary algebras and of canonical algebras, Adv. Math. 228 (2011), no. 3, 1405-1434, DOI 10.1016/j.aim.2011.06.016. MR2824559(2012h:16033)

[15] C. Chindris, On the invariant theory for tame tilted algebras, Algebra Number Theory 7 (2013), no. 1, 193-214, DOI 10.2140/ant.2013.7.193. MR.3037894

[16] C. Chindris, R. Kinser, and R. Weyman, Module varieties and representation type of finitedimensional algebras, Int. Math. Res. Notices, first published online October 16, 2013, DOI $10.1093 / \mathrm{imrn} / \mathrm{rnt} 216$.

[17] W. W. Crawley-Boevey and Jan Schröer, Irreducible components of varieties of modules, J. Reine Angew. Math. 553 (2002), 201-220, DOI 10.1515/crll.2002.100. MR.1944812 (2004a:16020)

[18] W. W. Crawley-Boevey, On tame algebras and bocses, Proc. London Math. Soc. (3) 56 (1988), no. 3, 451-483, DOI 10.1112/plms/s3-56.3.451. MR931510 (89c:16028)

[19] Corrado De Concini and Elisabetta Strickland, On the variety of complexes, Adv. in Math. 41 (1981), no. 1, 57-77, DOI 10.1016/S0001-8708(81)80004-7. MR625334(82m:14032)

[20] J. A. de la Peña, On the dimension of the module-varieties of tame and wild algebras, Comm. Algebra 19 (1991), no. 6, 1795-1807, DOI 10.1080/00927879108824229. MR.1113958 (92i:16016)

[21] Harm Derksen and Jerzy Weyman, Semi-invariants for quivers with relations, J. Algebra 258 (2002), no. 1, 216-227, DOI 10.1016/S0021-8693(02)00501-X. Special issue in celebration of Claudio Procesi's 60th birthday. MR 1958904 (2003m:16018)

[22] M. Domokos, Relative invariants for representations of finite dimensional algebras, Manuscripta Math. 108 (2002), no. 1, 123-133, DOI 10.1007/s002290200258. MR 1912950 (2003d:16017)

[23] M. Domokos, On singularities of quiver moduli, Glasg. Math. J. 53 (2011), no. 1, 131-139, DOI 10.1017/S0017089510000583. MR2747139 (2012a:16029)

[24] Piotr Dowbor and Andrzej Skowroński, On the representation type of locally bounded categories, Tsukuba J. Math. 10 (1986), no. 1, 63-72. MR846416 (88a:16057)

[25] Jean-Marc Drézet, Luna's slice theorem and applications, Algebraic group actions and quotients, Hindawi Publ. Corp., Cairo, 2004, pp. 39-89. MR2210794 (2006k:14082)

[26] Ju. A. Drozd, Tame and wild matrix problems (Russian), Representations and quadratic forms (Russian), Akad. Nauk Ukrain. SSR Inst. Mat., Kiev, 1979, pp. 39-74, 154. MR600111 (82m:16028)

[27] Christof Geiss and Jan Schröer, Varieties of modules over tubular algebras, Colloq. Math. 95 (2003), no. 2, 163-183, DOI 10.4064/cm95-2-2. MR1967418(2004d:16026)

[28] A. D. King, Moduli of representations of finite-dimensional algebras, Quart. J. Math. Oxford Ser. (2) 45 (1994), no. 180, 515-530, DOI 10.1093/qmath/45.4.515. MR1315461 (96a:16009)

[29] Witold Kraśkiewicz, On semi-invariants of tilted algebras of type $A_{n}$, Colloq. Math. 90 (2001), no. 2, 253-267, DOI 10.4064/cm90-2-5. MR.1876846 (2002m:16015)

[30] Witold Kraśkiewicz and Jerzy Weyman, Generic decompositions and semi-invariants for string algebras. Preprint available at arXiv:1103.5415, 2011.

[31] V. L. Popov and E. B. Vinberg, Algebraic geometry. IV, Encyclopaedia of Mathematical Sciences, vol. 55, Springer-Verlag, Berlin, 1994. Linear algebraic groups. Invariant theory; A translation of Algebraic geometry. 4 (Russian), Akad. Nauk SSSR Vsesoyuz. Inst. Nauchn. i Tekhn. Inform., Moscow, 1989 [ MR1100483 (91k:14001)]; Translation edited by A. N. Parshin and I. R. Shafarevich. MR 1309681 (95g:14002) 
[32] Z. Reichstein, On the notion of essential dimension for algebraic groups, Transform. Groups 5 (2000), no. 3, 265-304, DOI 10.1007/BF01679716. MR.1780933 (2001j:20073)

[33] Ch. Riedtmann, Tame quivers, semi-invariants, and complete intersections, J. Algebra 279 (2004), no. 1, 362-382, DOI 10.1016/j.jalgebra.2004.04.002. MR2078406 (2005j:16015)

[34] Ch. Riedtmann and G. Zwara, On the zero set of semi-invariants for tame quivers, Comment. Math. Helv. 79 (2004), no. 2, 350-361, DOI 10.1007/s00014-003-0797-2. MR2059437 (2005g:16024)

[35] Ch. Riedtmann and G. Zwara, The zero set of semi-invariants for extended Dynkin quivers, Trans. Amer. Math. Soc. 360 (2008), no. 12, 6251-6267, DOI 10.1090/S0002-9947-08-04613-8. MR2434286(2009i:14064)

[36] Claus Michael Ringel, The rational invariants of the tame quivers, Invent. Math. 58 (1980), no. 3, 217-239, DOI 10.1007/BF01390253. MR571574 (81f:16048)

[37] Maxwell Rosenlicht, Some basic theorems on algebraic groups, Amer. J. Math. 78 (1956), 401-443. MR0082183 (18,514a)

[38] Aidan Schofield, Birational classification of moduli spaces of vector bundles over $\mathbb{P}^{2}$, Indag. Math. (N.S.) 12 (2001), no. 3, 433-448, DOI 10.1016/S0019-3577(01)80020-3. MR.1914090 (2003g:14057)

[39] Daniel Simson and Andrzej Skowroński, Elements of the representation theory of associative algebras. Vol. 3, London Mathematical Society Student Texts, vol. 72, Cambridge University Press, Cambridge, 2007. Representation-infinite tilted algebras. MR 2382332 (2008m:16001)

[40] A. Skowroński and J. Weyman, The algebras of semi-invariants of quivers, Transform. Groups 5 (2000), no. 4, 361-402, DOI 10.1007/BF01234798. MR 1800533 (2001m:16017)

Department of Mathematics, University of Missouri-Columbia, Columbia, Missouri 65211

E-mail address: carrollat@missouri.edu

Current address: Department of Mathematical Sciences, DePaul University, Chicago, Illinois 60614

E-mail address: acarro15@depaul.edu

Department of Mathematics, University of Missouri-Columbia, Columbia, Missouri 65211

E-mail address: chindrisc@missouri.edu 